\title{
Ribosome actuator via the SpoT/ppGpp pathway to mitigate gene overexpression burden
}

\author{
Carlos Barajas $^{1}$, Jesse Gibson ${ }^{2}$, Luis Sandoval ${ }^{3}$, and Domitilla Del Vecchio ${ }^{1, *}$ \\ ${ }^{1}$ Department of Mechanical Engineering, Massachusetts Institute of Technology, Cambridge, Massachusetts \\ 2 Department of Bioengineering, Stanford University, Palo Alto, California \\ ${ }^{3}$ Department of Chemical Engineering, Massachusetts Institute of Technology, Cambridge, Massachusetts
}

* Correspondence: ddv@mit.edu

\begin{abstract}
Gene overexpression causes a burden on ribosomes, which decreases the expression of other genes and growth rate. Here, we engineer a genetic ribosome actuator that increases gene expression rate and growth rate by over $150 \%$ and $80 \%$, respectively. The actuator expresses the hydrolysis domain of the SpoT enzyme (SpoTH) in a bacterial strain with high basal levels of ppGpp, thus increasing ribosome availability. We use this actuator in a feedforward control arrangement, wherein SpoTH is coexpressed with a gene of interest (GOI). Without SpoTH co-expression, activation of the GOI decreases the expression of a constitutive gene and growth rate by over $80 \%$ and $40 \%$, respectively. By contrast, with SpoTH co-expression, the ribosome binding site (RBS) of SpoTH can be tuned to achieve inappreciable change of either the expression of the constitutive gene or growth rate when the GOI is activated to the same level. We finally employ the actuator to keep growth rate constant while expressing dCas9 to a level that would otherwise decrease growth rate by more than $40 \%$ due to toxicity. This actuator will be relevant in applications where high cellular protein concentrations are desired without growth defects or global reduction of gene expression.
\end{abstract}




\section{Introduction}

When a gene of interest (GOI) is activated in bacteria, cellular resources required for gene expression, chiefly ribosomes, are employed in the GOI's activation and thus are sequestered away from other genes. Increased burden on ribosomes, as the GOI is activated, leads to both decreased growth rate and reduced expression of other genes, synthetic or natural $[1,2,3,4]$. In a synthetic genetic circuit, this leads to coupling between the expression rates of otherwise independently regulated genes, which can completely destroy the intended circuit's function $[1,5,6]$. More generally, decreased growth rate leads to selective advantage of genetic mutants that disable the GOI's activation, which also leads to loss of function since mutants overtake the population $[7,8,9,10]$. Existing related works have reduced the burden on ribosomes upon expression of a GOI by reducing the GOI's expression rate itself to low levels [11]. These approaches result in low cellular concentrations of the GOI product, which are often not acceptable in genetic circuit design. In fact, higher concentrations of regulators and fluorescent reporters simplify genetic circuit composition, allow more accurate measurement of circuit output, and are critical in bacterial sensor applications $[12,13,14,15,16]$. Here, we seek a solution that allows high expression rates of a GOI with reduced burden on ribosomes. This will mitigate the purge on both growth rate and expression rate of any other gene while, at the same time, allowing activation of the GOI to a desired level as required, for example, in a synthetic genetic circuit. To the best of our knowledge, no such solution is available.

To this end, we leverage the SpoT/ppGpp pathway of E. coli to create a ribosome actuator. Ribosomes in E. coli are composed of three ribosomal RNAs (rRNA) and more than 50 proteins (r-proteins) [17]. Transcription of rRNA is the rate-limiting step in ribosome production [18, 19, 20,21, 22, 23, 24], while the concentration of r-proteins is autoregulated via translational inhibition to stoichiometrically match rRNA levels $[17,25,26,27]$. The production of rRNA is regulated by several protein factors $[28,29,30,31]$, and by the two (p)ppGpp analogs (ppGpp) [32,33]. During balanced exponential growth, ppGpp is the primary regulator of rRNA $[18,34,35]$ and there is an inverse relationship between basal ppGpp levels and both rRNA transcription and growth rate $[36,37,38,39]$. The RelA/SpoT Homologue (RSH) proteins are responsible for the synthesis and hydrolysis of ppGpp as shown in Fig. 1-a [40, 41, 42]. In particular, the SpoT enzyme is bifunctional with both synthesis and hydrolysis capabilities, with the latter dominating in exponential growth [43], while the RelA enzyme is monofunctional with solely synthesis activity. SpoT is responsible for maintaining basal ppGpp levels in steady state growth [44]. The actuator that we created controls exogenous expression of the hydrolysis domain of SpoT (SpoTH) in an exponentially growing bacterial strain that carries a mutation of the endogenous SpoT gene to render a higher basal ppGpp level [34, 36, 45, 46]. Activation of exogenous SpoTH thus catalyzes the hydrolysis of ppGpp [47], which upregulates both ribosome production and growth rate (Fig. 1-a).

We demonstrate that this actuator is capable of increasing the production rate of a constitutively expressed gene, a proxy for free ribosomes [2] (Supplementary Note 1) by more than $150 \%$ and growth rate by more than $80 \%$. We use this actuator to engineer a feedforward controller of ribosome concentration that maintains ribosome level approximately unchanged as the GOI is activated. This is achieved by co-expressing SpoTH with the GOI. Within this design, as the operon becomes activated, the expression of the GOI reduces free ribosome concentration, but expression of SpoTH also upregulates ribosome production through ppGpp hydrolysis. If the relative production rate between the GOI and SpoTH is properly tuned, then this ribosome upregulation can offset the decrease in free ribosome concentration, thereby maintaining ribosome level approximately constant. Activation of the GOI to its maximal level in the absence of the feedforward controller decreases the expression rate of a constitutive gene by more than $80 \%$ and growth rate by more than $40 \%$. By contrast, activation of the GOI to the same level in the presence of the feedforward controller shows inappreciable change in expression of the constitutive gene without decreasing growth rate, although growth rate increases by up to $40 \%$. We demonstrate that the RBS of SpoTH can be alternatively tuned to ensure no change in growth rate when the GOI is activated to the same level, while affecting the expression rate of the constitutive gene by less than $40 \%$.

Finally, we apply the ribosome actuator to overexpress dCas9 (as our GOI) without purging growth rate. dCas9 is toxic in many acteria $[48,49]$, which limits how highly it can be expressed, and low concentrations are sequestered away by sgRNAs, which hampers the function of CRISPRi-based circuits [12, 50]. We express dCas9 to levels that, otherwise, would reduce growth rate by more than $40 \%$. We therefore allow cellular concentrations of dCas9 that are at least four times the concentrations that are achievable with no growth defects without the ribosome actuator.

Overall, our ribosome actuator and feedforward controller allow high expression rate of a GOI with 
substantially reduced burden, thereby mitigating the purge on the expression rate of other genes and on growth rate.

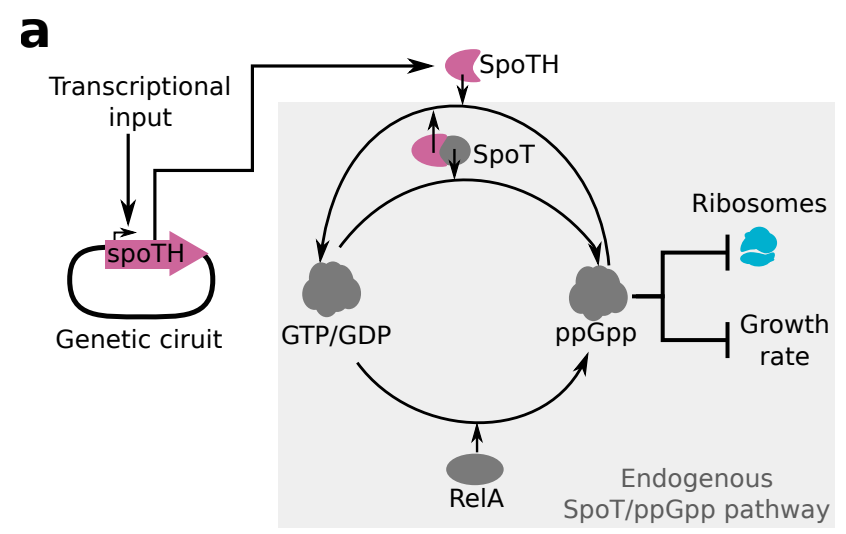

C

Desired actuator profile

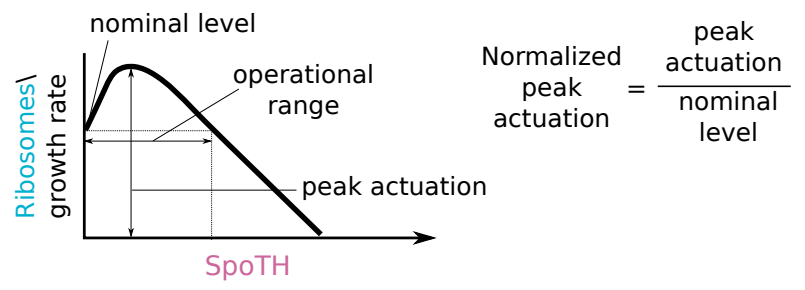

b

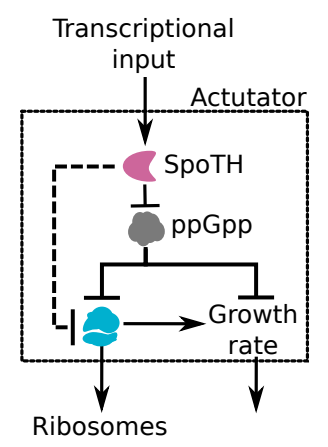

d

Strain with mutated spoT

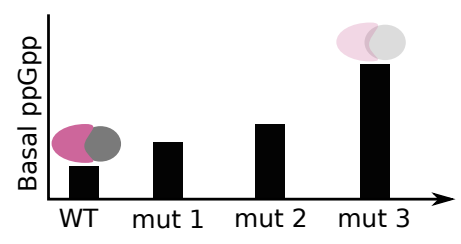

Figure 1: Actuating ribosome concentration and growth rate via SpoTH. (a) The nucleotide ppGpp negatively regulates ribosome production rate and growth rate in E. coli during exponential growth. The synthesis of ppGpp is catalyzed from GTP/GDP by RelA and by the synthesis domain of SpoT (in gray), while the hydrolysis of ppGpp is catalyzed by the hydrolysis domain of SpoT (in pink) [40, 41, 42]. Expression of the hydrolysis domain of SpoT (SpoTH) from a genetic circuit catalyzes the hydrolysis of ppGpp. (b) A transcriptional input activates SpoTH expression, which catalyzes the hydrolysis of ppGpp and hence upregulates both ribosome concentration and growth rate. Thus, SpoTH expression serves as a means to actuate both ribosome concentration and growth rate. The dashed edge between SpoTH and ribosomes represents sequestration of free ribosomes by SpoTH mRNA. (c) A desired actuator profile is one where ribosome concentration and growth rate initially increase as SpoTH is expressed. The expression of SpoTH mRNA continuously sequesters ribosomes, while the upregulation of ribosome production saturates when most of the ppGpp in the cell has been converted to GTP/GDP. We thus expect that the actuator profile will peak and then decrease after a certain amount of SpoTH expression. For a model explaining this expected actuator profile see Box 1. The nominal ribosome/growth rate level is the level when there is no SpoTH present. We define the peak ribosomal/growth rate level as the peak actuation and the SpoTH concentration where the actuator profile drops back to the nominal level as the operational range. The normalized peak actuation is defined as the peak actuation normalized by the nominal level. (d) Mutant bacterial strains are where the SpoT gene is mutated to provide several values of basal ppGpp level [36]. We denote the wild-type strain as WT and the three strains with mutated spoT as mut 1, mut2, and mut 3, respectively.

\section{Results}

\section{Actuating ribosome concentration and growth rate via SpoTH}

To actuate ribosome concentration and growth rate, we express SpoTH from a synthetic genetic circuit as shown in Fig. 1-a,b. The SpoTH gene only has ppGppase activity (see Supplementary note 3), which converts ppGpp to GTP/GDP but not vice-versa. A desired actuation profile of free ribosome concentration and growth rate as SpoTH is expressed, is one where both of these quantities increase as SpoTH concentration initially increases as shown in Fig. 1-c. Since the expression of SpoTH mRNA continuously sequesters ribosomes (see dashed line in Fig. 1-b), while the upregulation of ribosome production saturates when most of the ppGpp in the cell has been converted to GTP/GDP, we expect that the actuator profile will peak and then decrease after a certain amount of SpoTH expression. For a model of the expected actuator profile that captures this biphasic response (Fig 1-c), see Box 1.

Actuator profile in strains with varying basal ppGpp level. A cell with an elevated level of basal ppGpp has lower nominal ribosome/growth rate levels, but has a higher normalized peak actuation (Fig 1-c) since there is more ppGpp to act upon as a substrate in the SpoTH-catalyzed hydrolysis. Therefore, we 
characterize the actuation profile in three different strains carrying mutations in the SpoT gene resulting in different basal levels of ppGpp, as shown in Fig. 1-d. We test the CF944 (spoT202 allele), CF945 (spoT203 allele), and CF946 (spoT204 allele) strains, where the basal ppGpp levels are lowest for CF944 and highest for CF946 [36, 45, 46, 34]. Alongside these strains, we also characterize the actuation profile in the wild-type MG1655 strain (WT).

The genetic circuit used to express SpoTH in these strains is shown in Fig. 2-a,b. We increase the concentration of SpoTH by increasing the concentration of the aTc inducer and use the production rate of GFP as a proxy for free ribosome concentration (Supplementary note 1 and [51]). The data that shows the production rate of GFP and growth rate as SpoTH is induced in these different strains is shown in Fig. 2-c and Fig. 2-d, respectively. In SI Fig. 10 we show the GFP production rate and growth rate actuation in the DH10B strain (a commonly used lab strain [52]). We observe that neither GFP nor growth rate increase when SpoTH is expressed in DH10B. For MG1655 and CF944, we could actuate GFP production rate by $22 \%$ and $45 \%$, respectively (relative to $0 \mathrm{aTc}$ ), but were unable to positively actuate growth rate. For CF945, we can positively actuate GFP by $90 \%$ and growth rate by $80 \%$. For CF946, GFP initially decreases, but then increases by up to $65 \%$ and growth rate increases by $60 \%$. Therefore, CF945 is the only strain that has a desired actuation profile where both GFP production rate and growth rate increase as SpoTH expression increases and thus it is the strain we work with for the rest of this study. From Box 1, our simple mathematical model predicts that depending on the parameter regime, the actuation profile can: (I) have the qualitative behavior as in Fig. 1-c, (II) continuously decrease as SpoTH is increased (similar to the growth rates of MG1655 and CF944), (III) initially decrease and then increase (like the GFP production rate of CF946). According to our analysis in Box 1, having too little basal ppGpp (like in MG1655/CF944 [36]) or too much (like in CF946 [36]), can place the parameters outside the regime that renders the desired actuation profile. This model is consistent with our experimental findings that the strain CF945, with an intermediate concentration of basal ppGpp, renders the desired actuator profile. The GFP production rate and growth rate actuator profiles in Fig. 2-c,d do not fully correlate. For example, for CF945, GFP production rate peaks at aTc $\approx 215 \mathrm{nM}$, while growth rate continues to increase. This lack of correlation between GFP production rate and growth rate is expected since growth rate is regulated by an ensemble of endogenous proteins $[21,22,53,54]$ that are regulated directly by ppGpp [34, 55], while GFP is not regulated.

Tuning actuation profile via carbon source. The CF945 strain provides a desired actuation profile where both GFP production rate and growth rate increase as SpoTH is initially expressed. It has been shown that the growth medium composition, specifically carbon source, can determine the ppGpp basal level/nominal growth rate $[22,35,37,56,57]$. Therefore, we tune the nominal level and normalized peak actuation of the actuation profile (Fig. 1-b) in CF945, by varying the carbon source. We consider four common carbon sources used to support the growth of E. coli: glucose [1], glycerol [58], fructose [51], and lactose [59]. The GFP production rate normalized by the GFP production rate at aTc $=0 \mathrm{nM}$ (nominal GFP production rate) is shown in Fig. 2-e. The nominal GFP production rate vs the normalized peak GFP actuation for each carbon source is shown in Fig. 2-f. All carbon sources yield a desired actuation profile and there is an inverse relationship between the normalized peak GFP actuation and the nominal GFP production rate. For example, the nominal GFP production rate for fructose is 2.6 times higher than that of lactose, but in lactose, the GFP production rate can be actuated by up to $150 \%$ compared to just $50 \%$ for fructose. The growth rate normalized by the growth rate at aTc $=0 \mathrm{nM}$ (nominal growth rate) is shown in Fig. 2-g. The nominal growth rate vs the normalized peak growth rate actuation for each carbon source is shown in Fig. 2-h. For all carbon sources, a desired growth rate actuation profile is achieved. For glycerol, the nominal growth rate is $\approx 0.2 \mathrm{hr}^{-1}$ and growth rate can be increased by up to $\sim 85 \%$, while for glucose the nominal growth rate is $\approx 0.35 \mathrm{hr}^{-1}$, but growth rate can only be increased by up to $\sim 45 \%$. In the sequel, we focus solely on glycerol and lactose as carbon sources because actuation of both GFP production rate and growth rate is greatest in these two carbon sources. Between glycerol and lactose, we predominantly use glycerol since it provides a faster nominal growth rate and it is more commonly used.

The results from Fig. 2-f and Fig. 2-h show that there is a tradeoff between nominal GFP/growth rate levels and the normalized peak GFP/growth rate actuation. This tradeoff occurs because the actuation range is directly tied to the amount of ppGpp available to be hydrolyzed and high basal levels of ppGpp also reduce the baseline concentration of ribosomes. Our mathematical modeling (Box 1) supports this explanation as shown in Fig. 7 in Box 1. Also, as shown in the inset of Fig. 7 in Box 1, lower nominal levels lead to higher normalized peak actuation when the nominal levels are above a critical value. Below the critical value, normalized peak actuation decreases with nominal levels. This result is consistent with the experimental observation in Fig. 2-g, where the normalized peak growth actuation for lactose is lower than 
bioRxiv preprint doi: https://doi.org/10.1101/2021.02.11.430724; this version posted June 24, 2021. The copyright holder for this preprint (which was not certified by peer review) is the author/funder, who has granted bioRxiv a license to display the preprint in perpetuity. It is made available under aCC-BY-NC-ND 4.0 International license.

a P_GFP_SPOTH

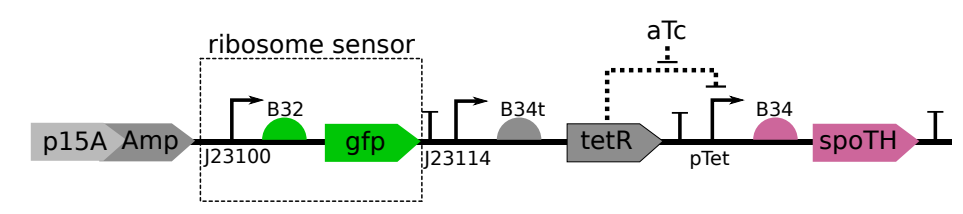

b

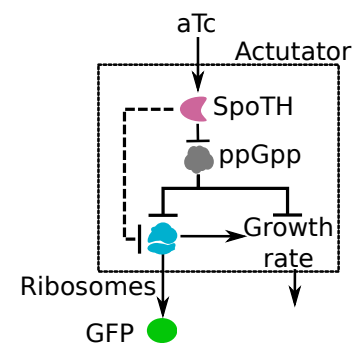

C

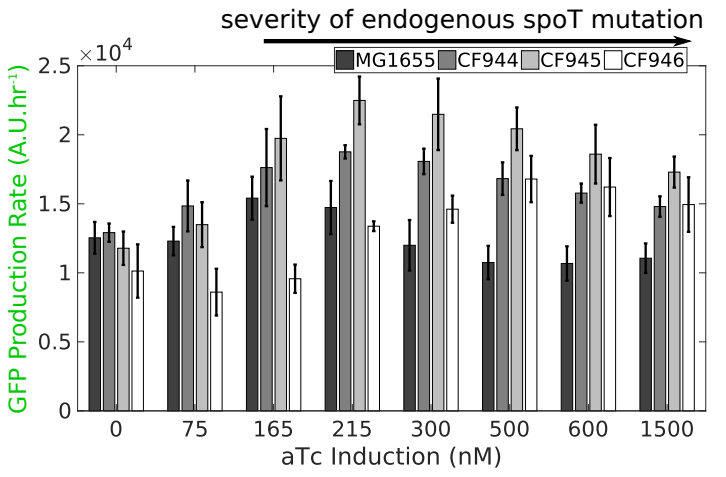

d

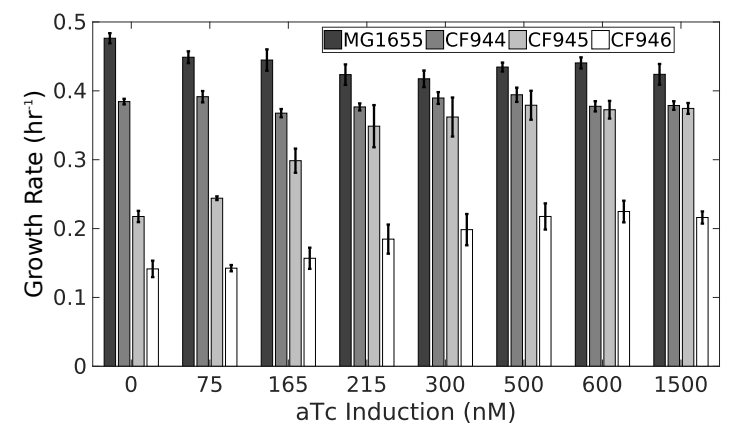

f

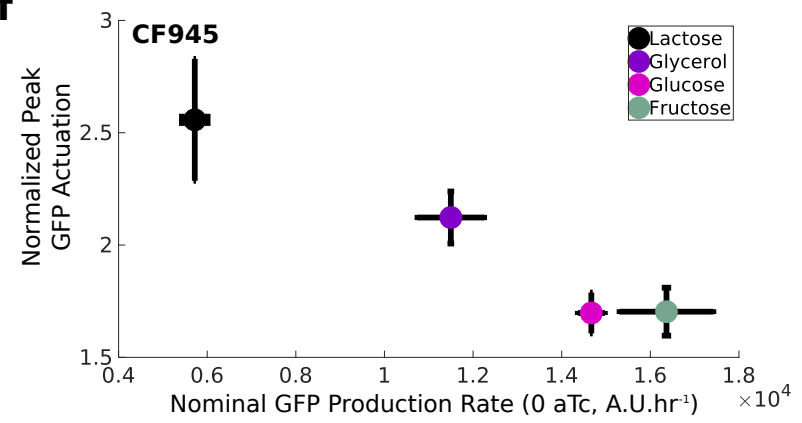

h

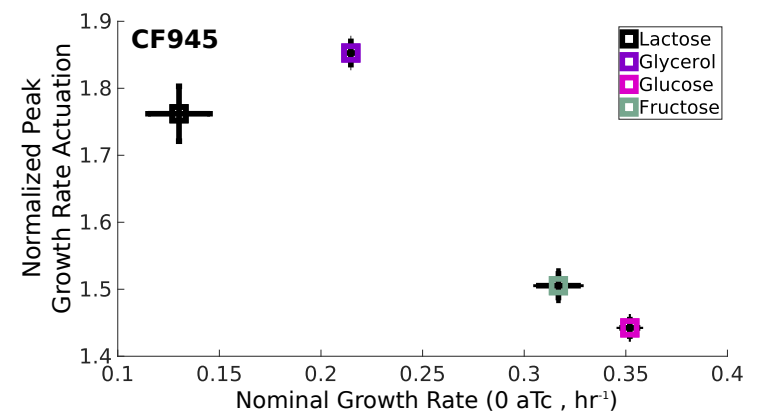

Figure 2: Bacterial strain and carbon source tune the actuation profile. (a) The P_GFP_SpoTH plasmid used to express SpoTH via the inducible pTet promoter. Plasmid description, plasmid map, and essential DNA sequences are provided in SI section Plasmid maps and DNA sequences. (b) Addition of aTc increases SpoTH concentration and thus upregulates both GFP production rate/free ribosome concentration and growth rate. (c)/(d) The GFP production rate/growth rate while increasing the expressing SpoTH in the wild-type MG1655, CF944, CF945, and CF946 strains [36] growing in glycerol as the sole carbon source. (e) The GFP production rate normalized by the GFP production rate at aTc $=0 \mathrm{nM}$, as SpoTH is expressed in CF945 growing in lactose, glycerol, fructose, or glucose as the sole carbon source. The normalized peak GFP actuation for each carbon source is marked by closed circles. The corresponding unnormalized data is shown SI Fig. 11-a. (f) The normalized peak GFP actuation versus the nominal GFP production rate for cells growing in each carbon source. (g) The growth rate normalized by the growth rate at aTc $=0 \mathrm{nM}$, as SpoTH is expressed in CF945 growing in lactose, glycerol, fructose, or glucose as the sole carbon source. The normalized peak growth rate actuation is marked by open squares. The corresponding unnormalized data is shown in SI Fig. 11-b. (h) The normalized peak growth rate actuation versus the nominal growth rate level for each carbon source. Data are shown as the mean \pm one standard deviation $(\mathrm{N}=4$, two biological replicates each with two technical replicates). The complete experimental protocol is provided in the Materials and Methods section. 
that in glycerol, even though lactose has a lower nominal growth rate.

\section{Using the actuator to mitigate burden on ribosomes due to heterologous gene overexpression}

Heterologous gene overexpression decreases the expression of all other genes, synthetic or natural, and growth rate through ribosome sequestration $[1,3,11]$. Furthermore, expression of proteins such as dCas9 decreases cell growth through toxicity [48, 49]. In many synthetic genetic circuit applications, it is critical to reach high cellular concentrations of a protein of interest, without substantially affecting growth rate nor impacting expression of other genes. For example, in CRISPRi-based genetic circuits, dCas9 should ideally be at high concentrations to minimize the effects of its sequestration by multiple sgRNAs [12, 50]. However, dCas9 is also toxic to the cell $[48,49]$, which has limited its concentration to ranges where sequestration effects are prominent. Similarly, high concentrations of both regulators' expression and fluorescent reporters in genetic circuits are desirable to simplify connection among genetic modules and to measure circuit function $[13,14]$. Most notably, fluorescent reporters used as outputs in bacterial biosensors are commonly expressed to high concentrations to ensure high output signal $[15,16]$. However, increased concentrations of one fluorescent reporter, reduces concentration of another one, thus impeding multiplexed sensing [1]. Here, we apply the ribosome actuator to enable high expression of a gene of interest, while mitigating the reduction of growth rate and global gene expression. To this end, we first assess the ability of the ribosome actuator to rescue protein production rates and growth rate as a red fluorescent protein (RFP) is overexpressed. Next, to automate the process of canceling the burden of expressing RFP, we demonstrate a feedforward controller in which SpoTH is co-expressed with RFP under the same inducible promoter. Finally, we demonstrate the application of the actuator to compensate for the toxic effects of dCas9 expression.

Actuator reduces burden caused by RFP overexpression. Here, we assess the ability of the SpoTH actuator to rescue the protein production rate of a constitutively expressed gene and growth rate when RFP is expressed. We expressed SpoTH and RFP under two independent inducible promoters as shown in Fig. 3a,b. Induction of RFP via AHL reduces ribosome availability, which, in turn, reduces the GFP production rate and growth rate (Fig. 3-c). Specifically, GFP production rate drops by up to $\sim 80 \%$ as RFP expression increases when there is no SpoTH expression (0 nM aTc). Expressing SpoTH shifts the relationship between GFP and RFP upwards, that is, more GFP is produced for a fixed RFP production rate. As a consequence, if we consider the nominal GFP production rate when there is no RFP nor SpoTH, then for every RFP production rate value up to $\approx 1.25 \times 10^{4}$ A.U.hr ${ }^{-1}$, we can find a SpoTH mRNA concentration that results in the same nominal GFP production rate. This result indicates that we can at least rescue a $60 \%$ decrease in GFP production rate caused by heterologous protein overexpression using the SpoTH actuator. Furthermore, for a fixed AHL induction, more RFP is produced when SpoTH is expressed than when it is not. These experimental results are consistent with the qualitative predictions from our mathematical model (Fig. 8 in Box 2). From Fig. 3-d, growth rate drops by up to $~ 35 \%$ as RFP expression increases when there is no SpoTH expression. Expressing SpoTH shifts also the relationship between growth rate and RFP production rate upwards. Therefore, for all RFP production rate values, there is a SpoTH level that yields a growth rate identical to the nominal growth rate. In Fig. 3-e, we plot the RFP production rate vs the aTc induction paths that keep GFP production rate and growth rate constant at their nominal values for all RFP production rates. The aTc induction path that keeps GFP production rate constant is not the same as the path that keeps growth rate constant. This is expected due to the lack of correlation between GFP production rate and growth rate as SpoTH is expressed (Fig. 2-c,d). As a comparison, SI Fig 12-a,b shows the GFP production rate and growth rate as RFP is expressed for several SpoTH levels in the WT strain. In this strain, when RFP is expressed without SpoTH expression, GFP production rate and growth rate drop by $80 \%$ and $20 \%$, respectively. SpoTH expression does not significantly increase GFP production rate nor growth rate.

Feedfoward controller to compensate for the burden from heterologous protein overepxression. The feedforward controller of ribosome concentration shown in Fig. 4-a is used to simultaneously express RFP and SpoTH. We refer to the open loop (OL) system when SpoTH is not present. We refer to the closed loop (CL) system when both RFP and SpoTH are expressed via the same TX input. In the CL system, when ribosomes are sequestered by RFP mRNA causing free ribosome concentration to decrease, SpoTH is also expressed and upregulates ribosome production, thus compensating for the ribosome sequestration. In Fig. 4-b, we show the expected OL response as RFP is expressed, the ideal response, and the expected CL response. The genetic circuit implementations of the OL and CL systems are shown in Fig. 4c,d. Our mathematical model shows that if the parameter regime is such that the actuator has a desired 
bioRxiv preprint doi: https://doi.org/10.1101/2021.02.11.430724; this version posted June 24, 2021. The copyright holder for this preprint (which was not certified by peer review) is the author/funder, who has granted bioRxiv a license to display the preprint in perpetuity. It is made available under aCC-BY-NC-ND 4.0 International license.
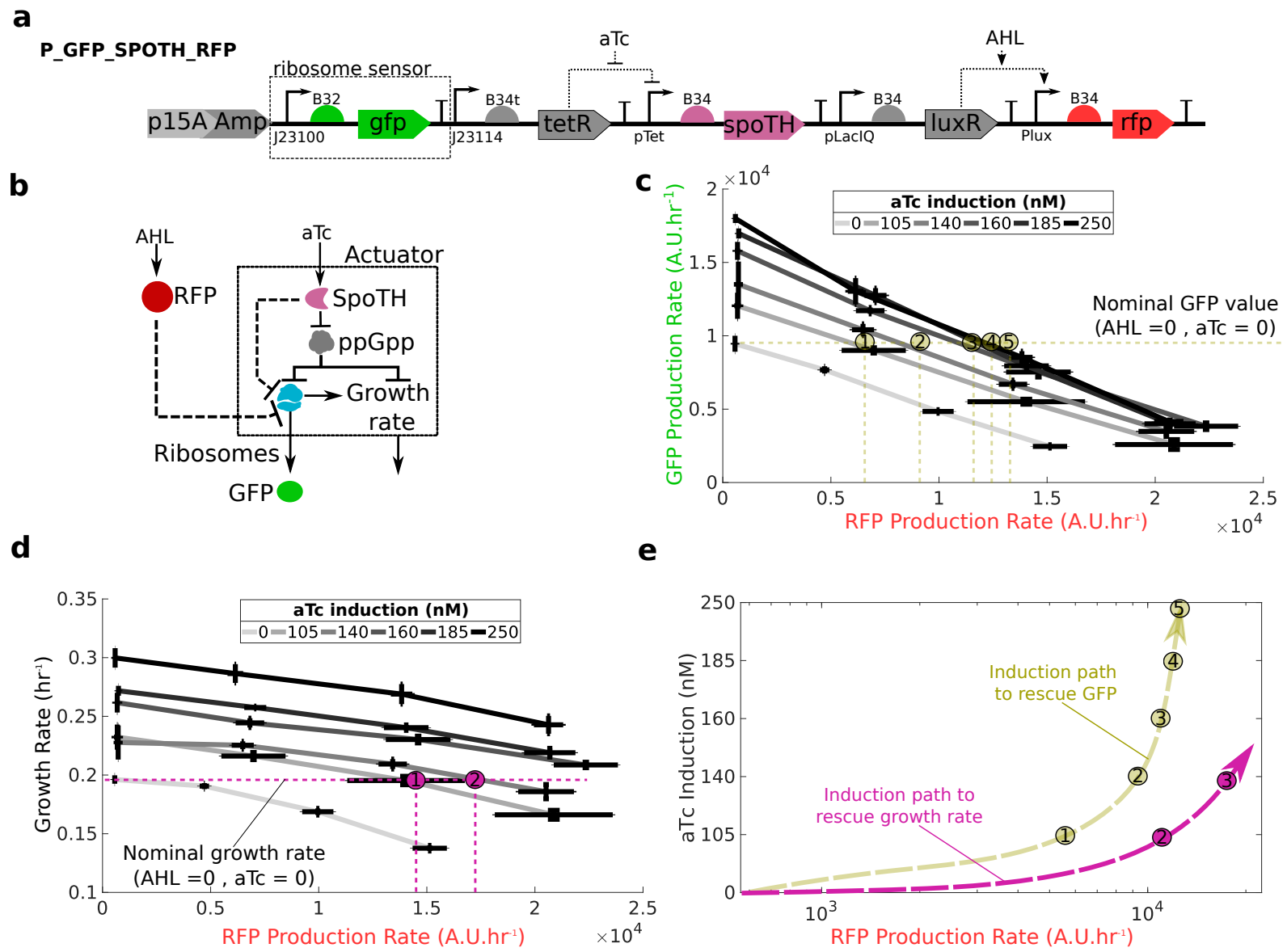

e

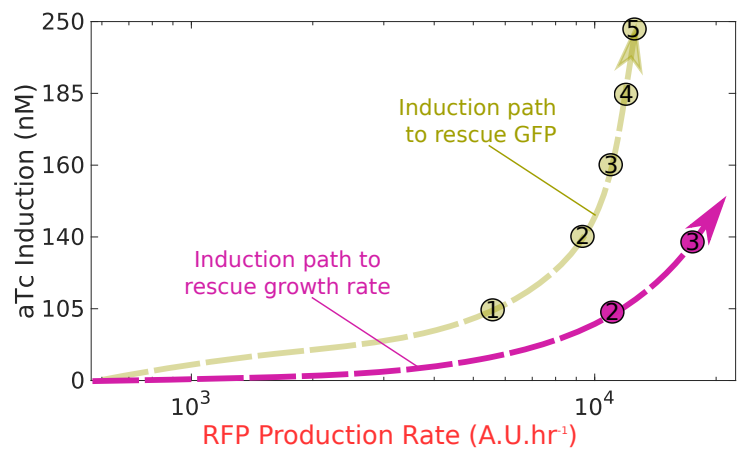

Figure 3: SpoTH actuator rescues protein production and growth rate reduced due to overexpressing a heterologous protein. (a) P_GFP_SpoTH_RFP plasmid used to express SpoTH using the inducible pTet promoter and RFP using the inducible Plux promoter. Plasmid description, plasmid map, and essential DNA sequences are provided in SI section Plasmid maps and DNA sequences. (b) AHL activates the production of RFP, which depletes free ribosome concentration by sequestering them for its translation, indicated by the dashed inhibition arrow from RFP to ribosomes. The addition of aTc increases SpoTH concentration, which actuates free ribosome concentration and growth rate by catalyizng the hydrolysis of ppGpp. SpoTH expression inhibits ribosomes by sequestration them for its translation, indicated by the dashed inhibition arrow from SpoTH to ribosomes. We use the production rate of a constitutively expressed GFP as a proxy for free ribosomes. (c) The GFP production rate as RFP production rate is increased, for different levels of SpoTH induction (denoted by shades of gray). The nominal GFP production rate corresponding to no RFP and no SpoTH expression (AHL $=0$ and aTc $=0$ ) is indicated by a horizontal yellow dashed-line. For each curve corresponding to a constant value of aTc, we denote with a vertical yellow dashed-line the RFP production rate that corresponds to the nominal GFP production rate. (d) Cell growth rate as RFP production rate is increased, for different levels of SpoTH induction (denoted by shades of gray). The nominal growth rate corresponding to no RFP and no SpoTH expression ( $\mathrm{AHL}=0$ and aTc $=0$ ) is indicated by a horizontal pink dashed-line. For each curve corresponding to a constant value of aTc, we mark with a vertical pink dashed-line the RFP production rate that yields the nominal growth rate. (e) aTc induction vs RFP production rate corresponding to a constant GFP production rate (yellow) or constant growth rate (pink). The paths are generated by linearly interpolating the data. Data are shown as the mean \pm one standard deviation ( $\mathrm{N}=4$, two biological replicates each with two technical replicates). All experiments were performed in the CF945 strain in media with glycerol as the sole carbon source. The AHL concentrations used in this experiment were AHL $=0 \mathrm{nM}, 3 \mathrm{nM}, 7 \mathrm{nM}, 15 \mathrm{nM}$. The complete experimental protocol is provided in the Materials and Methods section.

response (like CF945 in Fig. 2-c,d), then the SpoTH ribosome binding site (RBS) strength can be chosen such that the free ribosome concentration stays constant as RFP becomes expressed. We also consider a control genetic construct as shown in Fig. 4-e, where we replace SpoTH with a nonfunctional heterologous protein, CJB (cjBlue H197S [60]). This control construct allows to verify that the CL system performs better than the OL system due to the actuation properties of SpoTH and not because of the configuration change that the RFP mRNA undergoes when RFP is coexpressed with a second protein. We design four SpoTH RBS's to tune the CL system response (Supplementary note 2). In Fig. 4-f,g, we show the normalized GFP production rate/growth rate vs RFP production rate, when using glycerol as the carbon source. The OL system growth rate drops by over $40 \%$ when expressing RFP, while for the CL system with RBS 1 , the growth rate remains nearly constant when expressing RFP to a similar level as in the OL system. In SI Fig. 13 and Fig. 14, we 
show the unormalized AHL induction plots corresponding to Fig. 4-f,g. For the entire CL RBS library, the GFP production rates of OL and CL systems are nearly identical. The result from Fig. 2-f suggests that when using lactose as the carbon source, there is more GFP actuation than when using glycerol. Therefore, we tested the feedforward controller also when using lactose as the carbon source to better compensate for the reduction in GFP production rate as RFP is expressed. Fig. 4-h,i show the normalized GFP production rate/growth rate as RFP production rate increases for the control, OL, and CL systems, when using lactose as the carbon source. In the OL system, GFP production drops by more than $80 \%$ and growth rate by more than $40 \%$ when expressing RFP. In contrast, for the CL system with RBS 3, expressing RFP to the same level as in the OL system leads to an inappreciable change in GFP production rate without decreasing growth rate, although growth rate increases by up to $40 \%$. For the CL system with RBS 2, expressing RFP to the same level as in the OL system, leads to an inappreciable change in growth rate, while also reducing the decrease on GFP production rate to less than 40\%. In SI Fig. 15 and Fig. 16, we show the unormalized AHL induction plots corresponding to Fig. 4-h,i. As expected from Fig. 3-e, the RBS that best rescues GFP is not the same as the RBS that best rescues growth rate. In addition to compensating for growth rate and GFP production reduction, the CL system also allows increased production of RFP for each AHL level compared to the OL system. For all results in Fig. 4, the control performs worse than both the OL and CL systems as expected since we have the burden from both RFP and CJB without the SpoTH actuation. We conclude that when SpoTH is co-expressed with the GOI, the GOI can be expressed to levels higher than those reached without SpoTH while also mitigating the purge on growth rate and on global gene expression.

Actuator to reverse growth rate defects due to overexpressing a toxic protein. Here, we use the SpoTH actuator to reduce growth defects due to overexpressing the dCas9 protein, which is known to be toxic in many bacteria $[48,49]$. To this end, we express SpoTH using the inducible pTet promoter and dCas9 using the inducible Plux promoter as shown in Fig. 5-a. The induction of dCas9 with no SpoTH expression results in a $\sim 40 \%$ drop in growth rate, as shown in Fig. 5-b. Specifically, from Fig. 5-c (red data points), we observe that for the same production rates of RFP and dCas9, RFP elicits no decrease in growth rate while dCas9 does and up to $40 \%$. This indicates that the observed growth defects are likely due to direct toxicity as opposed to being due to ribosome sequestration, consistent with published literature $[61,62,12]$. Nevertheless, for every dCas9 induction level, there is a SpoTH induction that results in a growth rate that is near the nominal value when no dCas9 nor SpoTH are expressed (Fig. 5-b,c) Specifically, Fig. 5-c shows that for $\mathrm{AHL}=0.25 \mathrm{nM}$, growth rate drops by $\sim 8 \%$ when expressing dCas9 and without SpoTH, suggesting that toxicity is already present. However, by expressing $\mathrm{SpoTH}$, even for AHL $=2.0 \mathrm{nM}$, growth rate stays nearly constant. At AHL $=2.0 \mathrm{nM}$, nearly four times more RFP is produced than at $\mathrm{AHL}=0.25 \mathrm{nM}$. Under the assumption that RFP production rate is the same as dCas9 production rate, this implies that four times more dCas9 is produced at $\mathrm{AHL}=2.0 \mathrm{nM}$ than at $\mathrm{AHL}=0.25 \mathrm{nM}$. Thus, we conclude that with the appropriate SpoTH expression, we can produce four times the amount of dCas9 that would otherwise be toxic to the cell, while keeping growth rate constant. This factor of four may be conservative since from Fig. 3-b we observe that for a given AHL value more RFP is produced when SpoTH is induced. Therefore, we expect also that in Fig. 5-b, more dCas9 is produced when SpoTH is induced. SI Fig. 17-d shows that when dCas9 is expressed without expressing SpoTH, GFP production drops by $\sim 42 \%$. However, similar to growth rate, SpoTH expression can keep GFP production constant as dCas9 is expressed. This suggests that in addition to keeping growth rate constant when expressing dCas9, the SpoTH actuator aids in increasing the production rate of other genes while keeping growth rate unchanged.

\section{Discussion}

The alarmone ppGpp has been referred to as the "CEO of the cell", whose job is to optimally regulate resources for growth based on environmental conditions and current translational activity [54]. In this paper, we exploited the inverse relationship between ppGpp level and rRNA transcription during exponential growth $[36,37,38,39]$ and the hydrolysis of ppGpp by SpoT [43] to engineer an actuator that upregulates ribosome concentration. Specifically, the actuator exogenously expresses the hydrolysis domain of spoT (SpoTH) in a strain where the SpoT gene is mutated to render lower hydrolysis activity and hence higher ppGpp level (CF945). The CF945 strain grows about half as fast as the wild-type MG1655 strain (Fig 2-b and SI Fig. 12-b), however production rates are comparable in both strains (Fig 2-c and SI Fig. 12-a), implying that concentrations per cell (ratio between production rate and growth rate [63]) are not sacrificed when using the CF945 strain. We demonstrated that by changing the carbon source we can balance the tradeoff between nominal growth and maximal actuation, wherein higher maximal actuation corresponds to lower nominal 
bioRxiv preprint doi: https://doi.org/10.1101/2021.02.11.430724; this version posted June 24, 2021. The copyright holder for this preprint (which was not certified by peer review) is the author/funder, who has granted bioRxiv a license to display the preprint in perpetuity. It is made available under aCC-BY-NC-ND 4.0 International license.

a

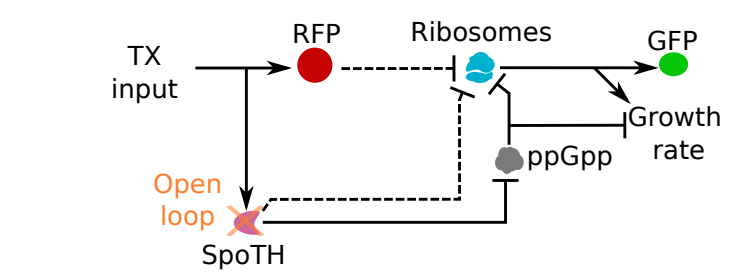

b

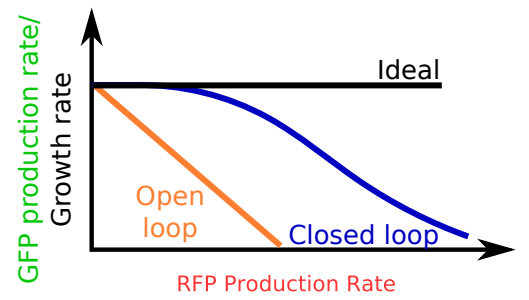

$\mathrm{AHL}$

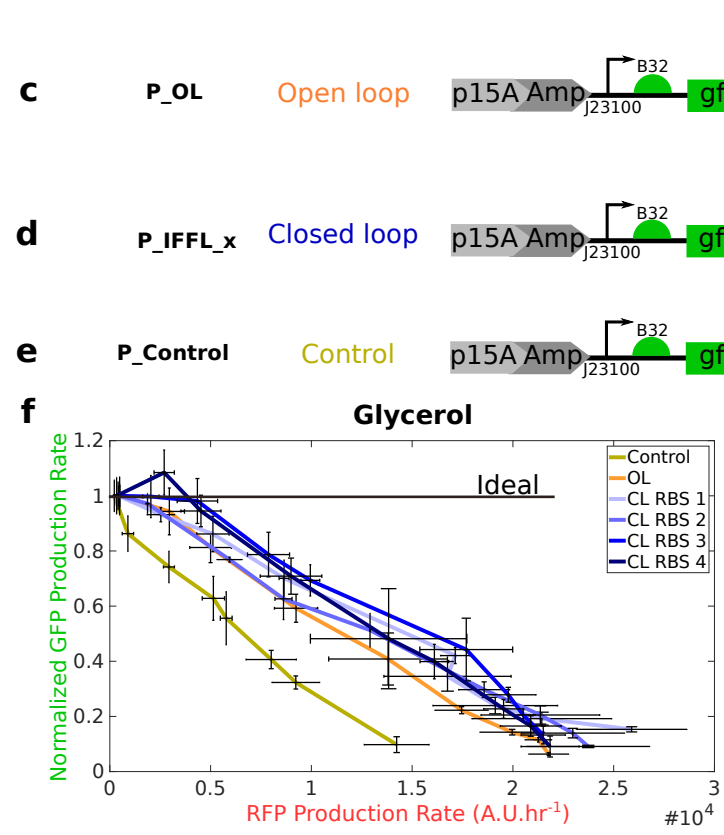

h

Lactose

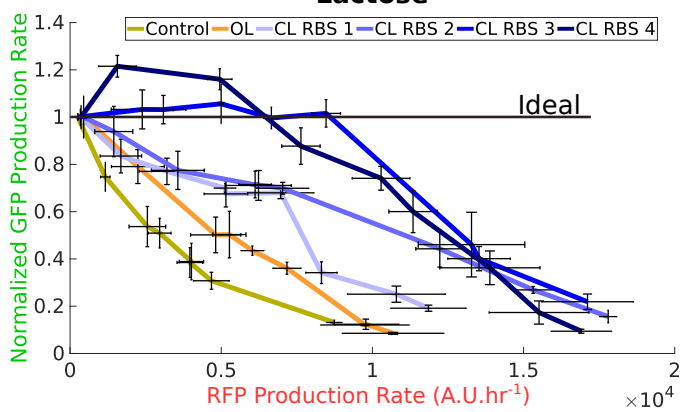

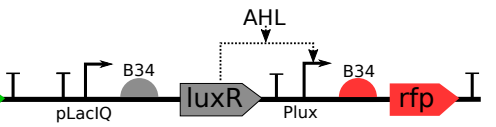

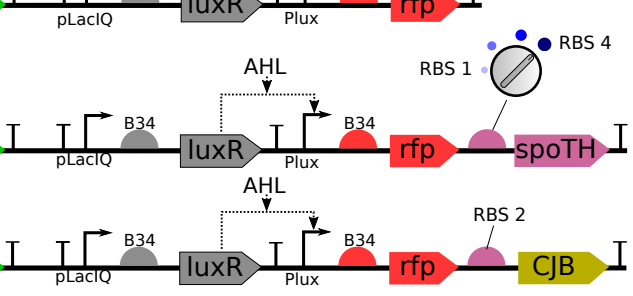

g
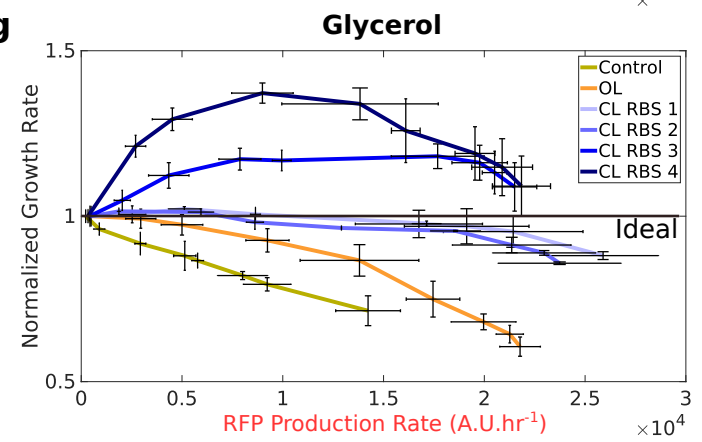

i

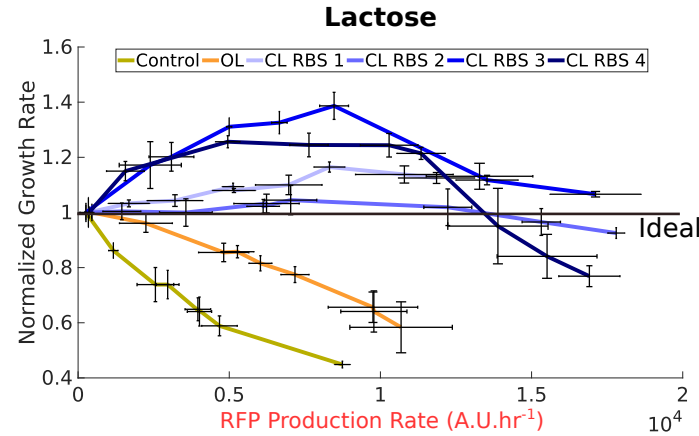

Figure 4: Feedforward controller to compensate for the burden on ribosomes caused by overexpressing RFP. (a) Diagram depicting the effect of expressing RFP (TX input) on ribosomes and growth rate for the open loop (OL) or closed loop (CL) systems. In the OL system, SpoTH is not present, so there is only the upper path from TX input to ribosomes. In the CL system, the TX input also activates SpoTH production and hence upregulates ribosome concentration and growth rate. Dashed edges represent sequestration of free ribosomes by a protein mRNA. (b) The expected GFP production rate/growth rate as RFP is expressed for the ideal case, the OL, and the CL systems. (c) OL system's genetic construct (P_OL) used to express RFP using the AHL (TX input) inducible Plux promoter (d) CL system's genetic construct (P_IFFL_x) used to simultaneously express RFP and SpoTH via the AHL inducible Plux promoter. The SpoTH RBS is used as a tuning parameter (see Model Box 1). (e) The control genetic construct (P_Control) used to simultaneously express RFP and CJB. The CJB protein is a nonfunctional heterologous protein. (f) Normalized GFP production rate normalized vs the RFP production rate for the control, OL, and CL systems (for different RBS's), using glycerol as the carbon source. (g) Normalized growth rate normalized by vs the RFP production rate for the control, OL, and CL systems (for different RBS's), using glycerol as the carbon source. (h) Normalized GFP production rate vs the RFP production rate for the control, OL, and CL systems (for different RBS's), using lactose as the carbon source. (i) Normalized growth rate vs the RFP production rate for the control, open loop, and closed loop (for 4 RBS), using lactose as the carbon source. Normalization is with respect to quantities at 0 AHL. Unnormalized data is provided in SI Fig. 13-SI Fig. 16 along with the exact AHL induction concentrations used. Data are shown as the mean \pm one standard deviation ( $\mathrm{N}=4$, two biological replicates each with two technical replicates). All experiments were performed in the CF945 strain. The complete experimental protocol is provided in the Materials and Methods section. Plasmid description, plasmid map, and essential DNA sequences are provided in SI section Plasmid maps and DNA sequences. 

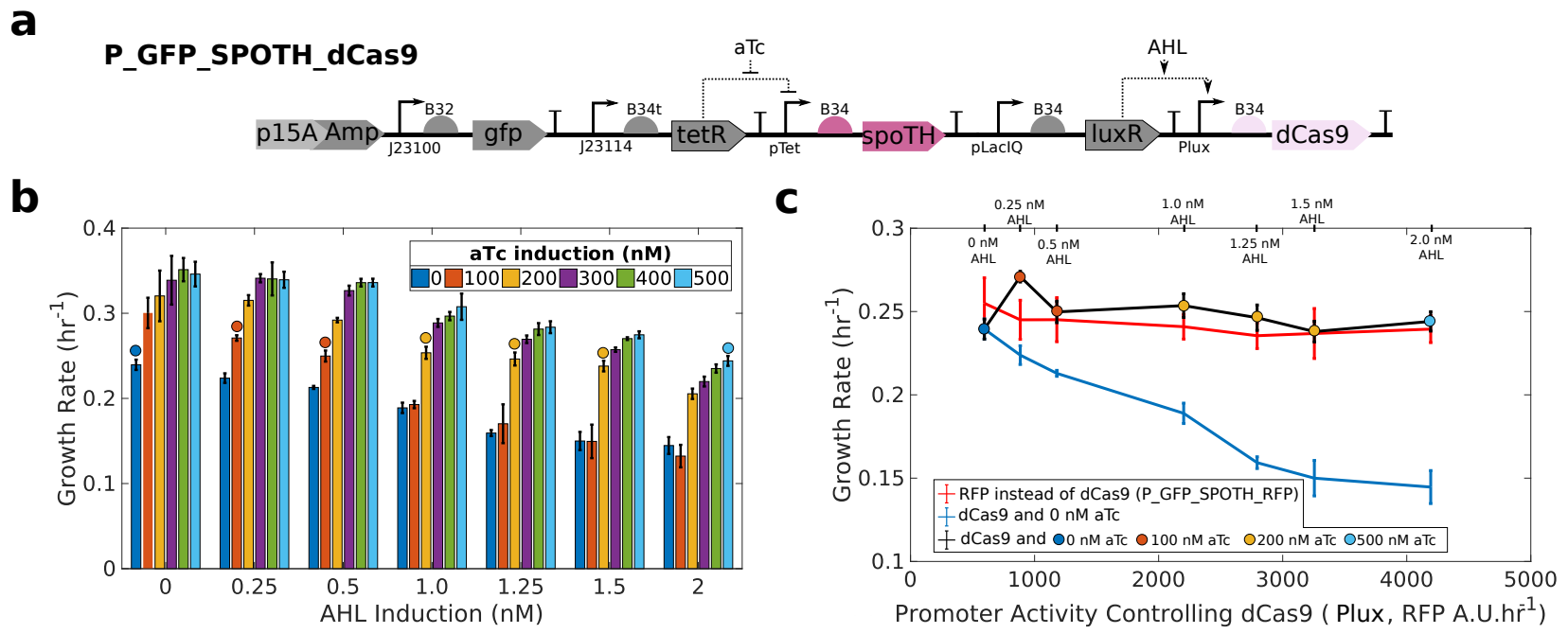

Figure 5: SpoTH actuator rescues growth rate reduction from dCas9 toxicity. (a) The P_GFP_SpoTH_dCas9 plasmid used to independently express SpoTH and dCas9. This construct is identical to the one in Fig. 3-a, but with the RFP coding sequence replaced by that of dCas9. Plasmid description, plasmid map, and essential DNA sequences are provided in SI section Plasmid maps and DNA sequences. (b) Cell growth rate as dCas9 production rate is increased via AHL, for different levels of SpoTH induction. For each AHL we mark by a circle the aTc level that results in a growth rate closets to the nominal growth rate when $\mathrm{AHL}=0 \mathrm{nM}$ and $\mathrm{aTc}=0 \mathrm{nM}$. (c) Cell growth rate vs Plux promoter activity as measured in units of RFP production rate (SI Fig. 17) for the same AHL induction levels as in (b) when Plux is transcribing RFP (red line, P_GFP_SpoTH_RFP with no SpoTH induction), dCas9 and with no SpoTH induction (blue line), and dCas9 with SpoTH induction corresponding to the colored circles in (b) (black line). Data are shown as the mean \pm one standard deviation (N=4, two biological replicates each with two technical replicates). All experiments were performed in the CF945 strain in media with glycerol as the sole carbon source. The complete experimental protocol is provided in the Materials and Methods section.

growth rate (Fig. 2-f,h). For example, using glucose as the carbon source, the actuator could upregulate growth rate by $45 \%$ and protein production rate by $60 \%$, and the cells had a doubling time of 2 hours (Fig. 2-e,g). By contrast, with lactose as the carbon source, the actuator could upregulate growth rate by $75 \%$ and protein production rate by $150 \%$, but the cells had a doubling time of 5 hours (Fig. 2-f,h). In this work, we choose to use the carbon source to tune the basal level of ppGpp, but other methods such as tuning the amino acid concentration in the media could also be considered [36].

We employed the actuator to create a feedforward controller that compensates for the burden from a GOI's overexpression and keeps free ribosome level approximately constant as the GOI is overexpressed (Fig. 4). The controller is realized by co-expressing SpoTH with the GOI (RFP). We tuned the controller's performance by changing the SpoTH RBS to guarantee practically no deviation in a constitutive gene's (GFP) expression rate as RFP's expression is increased (Fig. 4-h). However, for this RBS choice, growth rate increased by up to $40 \%$ (Fig. 4-i). We thus demonstrated that we can also choose an RBS such that practically no deviations in growth rate are observed when the GOI is expressed to the same level, with decreased purge on GFP expression rate (Fig. 4-h,i). As a final application, we considered dCas9 as our GOI. We showed that, with the SpoTH actuator, we could reach without growth defects a dCas9 production rate that would otherwise cause a $40 \%$ decrease in growth rate without SpoTH expression (Fig. 5-b). We estimate that this production rate is at least four times higher than that reachable without growth defects without SpoTH expression (Fig. 5-c).

Our understanding of ppGpp, and especially its role in exponential growth, is constantly evolving [54], so as we gain more insight on this pathway, we may uncover opportunities to further optimize the actuator. Furthermore, other enzymes like mesh1 [64, 65] and SpoT E319Q [66] have been shown to catalyze the hydrolysis of ppGpp and can, in principle, serve in alternative actuator designs.

Related work. Although not addressing the problem considered in this paper, other works considered the general problem of reducing the coupling among multiple genes' expression due to ribosome loading $[11,67,68]$. Specifically, in [11] a synthetic 16S rRNA was used to allocate a pool of "orthogonal" ribosomes to synthetic mRNAs while regulating the expression of $16 \mathrm{~S}$ rRNA via a feedback mechanism. This approach could, to some extent, decrease the coupling among genes due to (orthogonal) ribosome sharing, but to the price of fixing the RBS for all synthetic mRNA, thus removing a tuning parameter commonly used in the design of genetic circuits. In a different approach, a feedback controller was appended to a gene of 
interest to ensure that the GOI's production rate was robust to ribosome concentration variation due to gene overexpression burden $[69,68]$. However, these approaches were not designed to guarantee constant growth rate when a gene is overexpressed. More generally, the approaches in $[69,68]$ are suitable when the expression level of several genes is changing and we are interested in keeping a constant expression rate of a GOI. The approach of this paper is more suitable when we need to overexpress a GOI to reach high concentration per cell of the GOI's product, while keeping the expression rate of all other genes and growth rate constant. In future applications, the two approaches could be combined to obtain optimal solutions. In [12], dCas9 was re-engineered to allow overexpression without growth defects and loss in functionality. However, this modified dCas9 can only bind to promoters containing the the $30 \mathrm{bp}$ PhlF operator, thus making it not viable for genomic targets. Alternatively, a dCas9 regulator was engineered to neutralize competition in CRISPRi circuits by keeping a low but constant free level of dCas9 despite varying demand by sgRNAs [50]. The approach of this paper is complementary to this one and could be used in combination to both reach higher dCas9 levels without compromising growth and neutralize competition for dCas9.

Overall, this work provides an example of how we can exploit endogenous cell growth regulation pathways for engineering biology applications. The SpoTH actuator is a tool valuable in situations where high cellular concentrations of a protein of interest are desired, without substantially affecting growth rate or impacting expression of all other genes, such as in the design of synthetic genetic circuits and in their application. 
Here, we provide a simplified mathematical model that describes how expressing SpoTH actuates free ribosome concentration. The full model derivation and details can be found in SI Section: Derivation of the SpoTH actuator mathematical model. A key component of the model is the total ribosome concentration equation given by

$$
R_{T}=R+c_{s}+c_{e},
$$

where $R_{T}$ is the concentration of the total ribosomes in the cell, $R$ is the concentration of free ribosomes, and $c_{s}$ and $c_{e}$ are the concentrations of the mRNAribosome complex corresponding to SpoTH mRNA and the mRNA corresponding to the cell's endogenous genes, respectively. The concentration of SpoTH is proportional to $c_{s}$ (see corresponding SI section) and thus from hereon, we use varying SpoTH expression and varying $c_{s}$ interchangeably. Let $c_{e}$ be a general function of free ribosome concentration, that is, $c_{e}:=c_{e}(R)$. We assume that more endogenous mRNA is translated when $R$ increases, that is, $\frac{d c_{e}}{d R}>0$. We define

$$
z(R)=R+c_{e}(R),
$$

which satisfies $z(0)=0$ (in the absence of free ribosomes no endegnous mRNA is translated) and is monotonically increasing with $R$. We rewrite (1) using (2), using a model of how $R_{T}$ depends on $c_{s}$ through SpoTH catalyzing ppGpp hydrolysis (see corresponding SI section), and using overbars to denote concentrations normalized by the total ribosome concentration when there is no ppGpp in the cell, as:

$$
\bar{R}_{T}=\bar{z}_{0}+\left(1-\bar{z}_{0}\right) f\left(\bar{c}_{s} / \epsilon, \bar{z}_{0}\right)=\bar{z}+\bar{c}_{s},
$$

where $\bar{z}_{0} \in[0,1]$ is a proxy of the nominal free ribosome concentration corresponding to no SpoTH expression $\left(\bar{z}_{0}:=\left.\bar{z}\right|_{\bar{c}_{s}=0}\right), f \in[0,1)$ is given by $f=\frac{\left(\bar{c}_{s} / \epsilon\right)\left(\bar{c}_{s} / \epsilon+2\right)}{\left(\bar{c}_{s} / \epsilon\right)\left(\bar{c}_{s} / \epsilon+2\right)+1 / \bar{z}_{0}}$ and captures how SpoTH increases the total ribosome concentration, and $\epsilon$ is a dimensionless parameter that measures how effectively SpoTH catalyzes the hydrolysis of ppGpp and how effectively SpoTH-mRNA is translated into protein. An additional key quantity is

$$
\delta\left(\bar{z}_{0}, \epsilon\right):=\left.\frac{d \bar{z}}{d \bar{c}_{s}}\right|_{\bar{c}_{s}=0}=2 \frac{\bar{z}_{0}\left(1-\bar{z}_{0}\right)}{\epsilon}-1,
$$

which is the slope of $\bar{z}$ at $c_{s}=0$. The qualitative behavior of (3) is shown in Fig. 6 and it has three qualitatively different responses. When $\delta>0$, we obtain a desired actuator profile where $\bar{z}$ increases initially as $\bar{c}_{s}$ increases, as $\bar{c}_{s}$ continues to increase the $f$ term saturates to unity and the right hand side $\bar{c}_{s}$ term of (3) dominates and thus the actuator profile peaks and then decreases. As $\delta \rightarrow \infty$, the peak actuation and actuator operational range (as defined in
Fig 1-c) both approach the quantity $\left(1-\bar{z}_{0}\right)$. When $\delta<0, \bar{z}$ decreases initially as $\bar{c}_{s}$ increases and then it can either continue to decrease or it can eventually increase past $\bar{z}_{0}$, peak, and then decrease again.

Remark 1. From (4), for a fixed $\epsilon$ such that $\epsilon<0.5$, there exists $0<\bar{z}_{0, *}(\epsilon)<\bar{z}_{0}^{*}(\epsilon)<1$ such that for all $\bar{z}_{0} \in\left(\bar{z}_{0, *}, \bar{z}_{0}^{*}\right)$ we have that $\delta>0$ and for all $\bar{z}_{0}$ outside this set, $\delta<0$. In SI section: Derivation of the SpoTH actuator mathematical model (21), we show that $\bar{z}_{0}$ monotonically decreases with basal ppGpp and thus for a fixed $\epsilon$, there is an open interval of basal ppGpp values that render the desired actuation profile. This implies that too high or too low basal ppGpp can be detrimental in achieving the desired actuator profile.

In Fig. 7 , we show the normalized actuation $\left(\bar{z} / \bar{z}_{0}\right)$ profile for several $\bar{z}_{0}$ values. We observe that for lower $\bar{z}_{0}$ we have more normalized peak actuation. In the inset we show that the normalized peak actuation increases with $\bar{z}_{0}$ up until $\bar{z}_{0} \approx 0.1$. After this critical value, peak actuation decreases as $\bar{z}_{0}$ decreases.

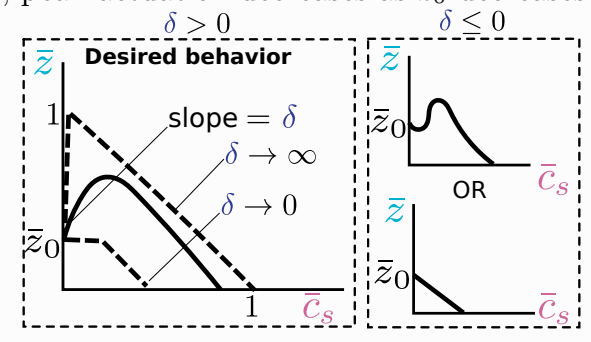

Figure 6: Qualitative behavior of actuator. For $\delta>0(4)$, the actuator profile predicted by (3) has the desired behavior where $\bar{z}$ (proxy of free ribosomes) increases as SpoTH is expressed (increasing $\bar{c}_{s}$ ), then it peaks and begins to drop. The asymptotic behavior as $\delta \rightarrow \infty$ and $\delta \rightarrow 0$ are depicted by dashed lines. When $\delta<0, \bar{z}$ initially decreases as SpoTH is expressed. It can then either continue to decrease or at some point increase, peak, and then decrease again.

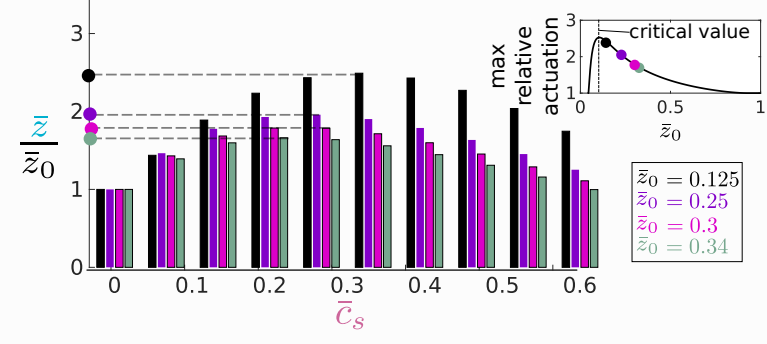

Figure 7: Tradeoff between nominal level and normalized peak actuation. The actuation profile predicted by (3) for several $\bar{z}_{0}$. We observe that for lower $\bar{z}_{0}$, there is higher normalized peak actuation. In the inset we show that the normalized actuation increases as $\bar{z}_{0}$ increases up until the critical value of $\bar{z}_{0} \approx 0.1$. After this critical value normalized peak actuation decreases as $\bar{z}_{0}$ increases. For this simulation we have $\epsilon=0.13$. 
bioRxiv preprint doi: https://doi.org/10.1101/2021.02.11.430724; this version posted June 24, 2021. The copyright holder for this preprint (which was not certified by peer review) is the author/funder, who has granted bioRxiv a license to display the preprint in perpetuity. It is made available under aCC-BY-NC-ND 4.0 International license.

Box 2 Actuator to cancel burden from heterologous protein overexpression

When accounting for the expression of a heterologous protein $\mathrm{y}$, the total ribosome concentration equation (1) is modified to

$$
R_{T}=R+c_{s}+c_{y}+c_{e}
$$

where $c_{y}$ is the concentration of the mRNA-ribosome complex corresponding to the mRNA of $y$. The protein concentration of $\mathrm{y}$ is proportional to $c_{y}$. The dimensionless total ribosome equation (3) now reads

$$
\bar{z}_{0}+\left(1-\bar{z}_{0}\right) f\left(\bar{c}_{s} / \epsilon, \bar{z}_{0}\right)=\bar{z}+\bar{c}_{s}+\bar{c}_{y} .
$$

In SI Section: Derivation of the SpoTH actuator mathematical model, we show that at steady state, the quantities $\bar{c}_{y}$ and $\bar{c}_{s}$ are given by

$$
\bar{c}_{y}=m_{y} \bar{R} / K_{y}, \quad \bar{c}_{s}=m_{s} \bar{R} / K_{s},
$$

where $m_{y}$ is the y mRNA concentration, $K_{y}$ is the dissociation constant of free ribosome with y mRNA, $m_{s}$ is the SpoTH mRNA concentration, and $K_{s}$ is the dissociation constant of free ribosome with SpoTH mRNA. Each of $K_{y}$ and $K_{s}$ can be tuned by changing the ribosome binding site (RBS) of the corresponding mRNA. In Fig. 8, we show that increasing the concentration of y $\left(\bar{c}_{y}\right)$ when no SpoTH is expressed $\left(m_{s}=0\right)$ causes $\bar{z}$ to drop by $80 \%$. Expressing SpoTH shifts the relationship between $\bar{z}$ and $\bar{c}_{y}$ upwards. Therefore, for a fixed $\bar{c}_{y}$ there corresponds a SpoTH level $\left(\bar{c}_{s}\right)$ that renders $\bar{z}=\bar{z}_{0}$. Also, for a fixed $m_{y}$, increasing SpoTH expression increased $\bar{c}_{y}$ and thus the amount of y produced. From (7), we have that

$$
\bar{c}_{s}=\frac{m_{s}}{m_{y}} \gamma \bar{c}_{y}, \quad \gamma=K_{y} / K_{s},
$$

where $\gamma$ is the ratio between the SpoTH RBS strength and the y RBS strength.

Feedforward controller We model SpoTH and y as being transcribed from the same promoter, which implies $m_{s}=m_{y}$. We refer to the configuration where y and SpoTH are transcriptionally coupled this way as the closed loop system and it obeys (6) with $\bar{c}_{s}=\gamma \bar{c}_{y}$ as shown by (8) when $m_{s}=m_{y}$. We denote expressing $\mathrm{y}$ in the absence of SpoTH as the open loop system and it obeys (6) with $\bar{c}_{s}=0$ for all values of $\bar{c}_{y}$. The qualitative behavior of the closed loop system compared to the open loop system is shown in SI Fig. 18. We define the ideal relationship between $\bar{z}$ and $\bar{c}_{y}$ as $\bar{z}=\bar{z}_{0}$ for all $\bar{c}_{y}$, as shown in Fig. 9 . The initial slope $\left(\left.\frac{d \bar{z}}{d \bar{c}_{y}}\right|_{\bar{c}_{y}=0}\right)$ of the closed loop system is given by $\gamma \delta-1$, where $\delta$ is given by (4). Thus, if $\delta>0$, which, from Fig 6, implies that we are in the parameter regime such that the actuator has a desired profile, then the SpoTH RBS strength $(\gamma)$ can be chosen such that $\gamma\left(\bar{z}_{0}, \epsilon\right)=1 / \delta\left(\bar{z}_{0}, \epsilon\right)$ to render an initial flat response of $\bar{z}$ as $\bar{c}_{y}$ increases. In Fig. 9, we show the closed loop system response (blue lines) for $\gamma<1 / \delta, \gamma=1 / \delta$, and $\gamma>1 / \delta$ and the open loop system response (orange). As expected, for $\gamma=1 / \delta$, the response of $\bar{z}$ is initially flat as $\bar{c}_{y}$ is expressed. The closed loop system achieves higher values of $\bar{z}$ than the open loop system. Furthermore, we observe that the closed loop system achieves higher values of $c_{y}$ than the open loop system.

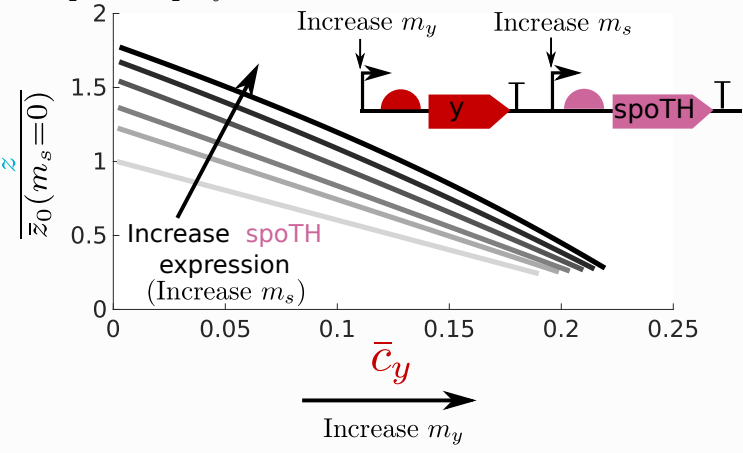

Figure 8: SpoTH expression rescues free ribosomes reduced due to heterologous protein overexpression. Simulation of (6) using (7) when y mRNA $\left(m_{y}\right)$ and SpoTH mRNA $\left(m_{s}\right)$ are varied independently. Increasing $m_{y}$ and thus $\bar{c}_{y}$, causes $\bar{z}$ (proxy for free ribosomes) to drop. Expressing SpoTH shifts the relationship between $\bar{z}$ and $\bar{c}_{y}$ upwards. The max $m_{y}$ simulated is fixed for all $m_{s}$ levels, however the max $\bar{c}_{y}$ achieved increases with $m_{s}$. For this simulation we have $\bar{z}_{0}=0.25$ and $\epsilon=0.13$. See Supplementary Note 4 for additional simulation details.

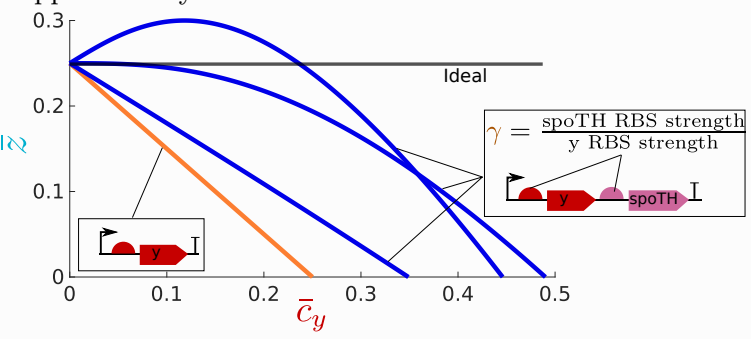

Figure 9: Feedforward controller to compensate for the burden on ribosomes caused by heterologous protein overexpression. Simulation of (6) with $\bar{c}_{s}=\gamma \bar{c}_{y}$. This corresponds to placing y and SpoTH under same promoter (closed loop) depicted in blue. The SpoTH RBS $(\gamma)$ can be tuned to approximate the ideal scenario where $\bar{z}=\bar{z}_{0}$ for all $\bar{c}_{y}$. We also show the open loop system (y without SpoTH) depicted in orange as given by (6) with $c_{s}=0$. For this simulation we have $\bar{z}_{0}=0.25$ and $\epsilon=0.13$. For the closed loop, we have that $\gamma=0.16,0.53,0.9$.

\section{Materials and Methods}

Bacterial strain and growth. The bacterial strain used for genetic circuit construction was E. coli NEB10B (NEB, C3019I) and LB broth Lennox was the growth medium used during construction. Characterization was per- 
formed using the CF944, CF495, and CF946 [36], MG1655 (CGSC, 6300), and DH10B [52] strains. Characterization experiments were done using M9 minimal medium supplemented with $0.2 \%$ casamino acids, 1 mM thiamine hydrochloride, ampicillin $(100 \mu \mathrm{g} / \mathrm{mL})$, and either $0.4 \%$ glucose, $0.4 \%$ fructose, $0.4 \%$ glycerol, or $2 \mathrm{~g} / \mathrm{L}$ lactose (the specific carbon source used for each experiment is specified in the figure caption).

Microplate photometer protocol. Cultures were prepared by streaking cells from a $15 \%$ glycerol stock stored at $-80^{\circ} \mathrm{C}$ onto a $\mathrm{LB}$ (Lennox) agar plate containing $100 \mu \mathrm{g} / \mathrm{mL}$ ampicillin and incubated at $37^{\circ} \mathrm{C}$. Isolated colonies were picked and grown in $2 \mathrm{ml}$ of growth medium in culture tubes (VWR, 60818-667) for $12-24$ hours at $30^{\circ} \mathrm{C}$ and $220 \mathrm{rpm}$ in an orbital shaker. Cultures were then diluted to an $\mathrm{OD}$ at $600 \mathrm{~nm}\left(\mathrm{OD}_{600 \mathrm{~nm}}\right)$ of 0.0075 and grown for an additional 6 hours in culture tubes to ensure exponential growth before induction. Cultures were then induced and plated onto 96 well-plate (Falcon, 351172). The 96 -well plate was incubated at $30^{\circ} \mathrm{C}$ in a Synergy MX (Biotek, Winooski, VT) microplate reader in static condition and was shaken at a fast speed for $3 \mathrm{~s}$ right before OD and fluorescence measurements. Sampling interval was 5 minutes. Excitation and emission wavelengths to monitor GFP fluorescence are 485 and $530 \mathrm{~nm}$, respectively and those to monitor RFP fluorescence are 584 and $619 \mathrm{~nm}$, respectively. Sampling continued until bacterial cultures entered the stationary.

Calculating growth rate and protein production rates. The media background OD $\left(0.08 \mathrm{OD}_{600 \mathrm{~nm}}\right)$ and GFP (100 AU) were subtracted from the data prior to any calculations. To ensure the data analyzed was coming from cells in exponential growth, only OD values (adjusted for background) of $\mathrm{OD}_{600 \mathrm{~nm}}=0.06$ and $\mathrm{OD}_{600 \mathrm{~nm}}=0.14$ were considered except for experiments done in lactose were the range was $\mathrm{OD}_{600 \mathrm{~nm}}=0.06$ and $\mathrm{OD}_{600 \mathrm{~nm}}=0.1$, since cells growing in lactose entered stationary phase at lower OD values.

To dampen noise before differentiating, the data was then filtered using a moving average filter. Given a signal with $n$ measurements $\mathbf{y}=\left[y_{1}, y_{2}, \ldots, y_{n+1}\right]$ sampled at a constant period $\Delta t$, we apply the moving average filter as follow:

$$
d_{j}=\sum_{r=-2}^{2} \frac{y_{j+r}}{5}, \quad \forall j \in[3,4, \ldots, n-1],
$$

where $\mathbf{d}=\left[d_{1}, d_{2}, \ldots, d_{n+1}\right]$ is our filtered signal with boundary points identical to those of $\mathbf{y}\left(d_{1}=y_{1}\right.$ and $\left.d_{2}=y_{2}\right)$.

The growth rate $\mu$ is calculated from the filtered OD signal by performing linear regression (in a least-squares sense) on the log of the signal and taking the slope of the fit. The RFP and GFP production rates were calculated in a similar manner as [51]. Denoting $\operatorname{GFP}\left(t_{i}\right)$ and $\operatorname{RFP}\left(t_{i}\right)$ as the filtered GFP and RFP signal measured by the plate reader at time $t_{i}$, the GFP production rate $\left(\alpha_{\mathrm{GFP}}\left(t_{i}\right)\right)$ and $\mathrm{RFP}$ production rate $\left(\alpha_{\mathrm{RFP}}\left(t_{i}\right)\right)$ are given by

$$
\alpha_{\mathrm{GFP}}\left(t_{i}\right)=\frac{\operatorname{GFP}\left(t_{i+1}\right)-\operatorname{GFP}\left(t_{i-1}\right)}{2\left(t_{i+1}-t_{i-1}\right) \mathrm{OD}\left(t_{i}\right)}, \quad \alpha_{\mathrm{RFP}}\left(t_{i}\right)=\frac{\operatorname{RFP}\left(t_{i+1}\right)-\operatorname{RFP}\left(t_{i-1}\right)}{2\left(t_{i+1}-t_{i-1}\right) \mathrm{OD}\left(t_{i}\right)},
$$

where $\mathrm{OD}\left(t_{i}\right)$ is the filtered OD level.

\section{Genetic circuit construction.}

The genetic circuit construction was based on Gibson assembly [70]. DNA fragments to be assembled were amplified by PCR using Phusion High-Fidelity PCR Master Mix with GC Buffer (NEB, M0532S), purified with gel electrophoresis and Zymo clean Gel DNA Recovery Kit (Zymo Research,D4002), quantified with the nanophotometer (Implen, P330), and assembled withGibson assembly protocol using NEBuilder HiFi DNA Assembly Master Mix(NEB, E2621S). Assembled DNA was transformed into competent cells prepared by the CCMB80 buffer (TekNova, C3132). Plasmid DNA was prepared by the plasmid miniprep-classic kit (Zymo Research, D4015). DNA sequencing used Quintarabio DNA basic sequencing service. Primers and gBlocks were obtained from Integrated DNA Technologies. The list of constructs and essential DNA sequences can be found in SI section Plasmid maps and DNA sequences

\section{Acknowledgements}

This work was supported in part by NSF Expeditions, Grant Number 1521925, AFOSR grant FA9550-14-1-0060, the NSF Graduate Research Fellowships Program, and the Ford Foundation Predoctoral Fellowship. We thank Hsin-Ho Huang for helping guide the plasmid construction. We thank Dr. Cashel, Dr. Potrykus, and Dr. Fernández-Coll for providing the CF944, CF945, and CF946 strains and their helpful discussion on ppGpp.

\section{Author contributions}

D.D.V. and C.B designed the study; J.G, L.S, and C.B built the genetic circuits; C.B performed the experiments; C.B analyzed the data; C.B developed the mathematical models; C.B and D.D.V. wrote the paper. 


\section{Competing interests}

The authors declare that there is no conflict of interest.

\section{References}

[1] A. Gyorgy, J. I. Jiménez, J. Yazbek, H. H. Huang, H. Chung, R. Weiss, and D. Del Vecchio, "Isocost Lines Describe the Cellular Economy of Genetic Circuits," Biophys. J., vol. 109, no. 3, pp. 639-646, 2015.

[2] F. Ceroni, R. Algar, G.-B. Stan, and T. Ellis, "Quantifying cellular capacity identifies gene expression designs with reduced burden," Nat. Methods, vol. 12, no. 5, pp. 415-418, 2015.

[3] I. Shachrai, A. Zaslaver, U. Alon, and E. Dekel, "Cost of Unneeded Proteins in E. coli Is Reduced after Several Generations in Exponential Growth," Mol. Cell, vol. 38, no. 5, pp. 758-767, 2010.

[4] M. S. Bienick, K. W. Young, J. R. Klesmith, E. E. Detwiler, K. J. Tomek, and T. A. Whitehead, "The interrelationship between promoter strength, gene expression, and growth rate," PLoS One, vol. 9, no. 10, 2014.

[5] J. Vind, M. A. Sørensen, M. D. Rasmussen, and S. Pedersen, "Synthesis of proteins in Escherichia coli is limited by the concentration of free ribosomes. Expression from reporter genes does not always reflect functional mRNA levels.," 1993.

[6] Y. Qian, H. H. Huang, J. I. Jiménez, and D. Del Vecchio, "Resource Competition Shapes the Response of Genetic Circuits," ACS Synth. Biol., vol. 6, no. 7, pp. 1263-1272, 2017.

[7] C. W. Johnston and J. J. Collins, "Engineering microbial peer pressure," Science (80-. )., vol. 365, no. 6457, pp. 986-987, 2019.

[8] M. Manhart and E. I. Shakhnovich, "Growth tradeoffs produce complex microbial communities on a single limiting resource," Nat. Commun., vol. 9, no. 1, 2018.

[9] N. S. McCarty and R. Ledesma-Amaro, "Synthetic Biology Tools to Engineer Microbial Communities for Biotechnology," 2019.

[10] L. Pacciani-Mori, S. Suweis, A. Maritan, and A. Giometto, "Constrained proteome allocation affects coexistence in models of competitive microbial communities," ISME J., 2021.

[11] F. Ceroni, A. Boo, S. Furini, T. E. Gorochowski, O. Borkowski, Y. N. Ladak, A. R. Awan, C. Gilbert, G. B. Stan, and T. Ellis, "Burden-driven feedback control of gene expression," Nat. Methods, vol. 15, no. 5, pp. 387-393, 2018 .

[12] S. Zhang and C. A. Voigt, "Engineered dCas9 with reduced toxicity in bacteria: Implications for genetic circuit design," Nucleic Acids Res., vol. 46, no. 20, pp. 11115-11125, 2018.

[13] J. A. N. Brophy and C. A. Voigt, "Principles of genetic circuit design," Nat. Methods, vol. 11, no. 5, pp. 508-520, 2014.

[14] T. E. Gorochowski, A. Espah Borujeni, Y. Park, A. A. Nielsen, J. Zhang, B. S. Der, D. B. Gordon, and C. A. Voigt, "Genetic circuit characterization and debugging using RNA -seq," Mol. Syst. Biol., vol. 13, no. 11, p. 952, 2017.

[15] K. N. Daeffler, J. D. Galley, R. U. Sheth, L. C. Ortiz-Velez, C. O. Bibb, N. F. Shroyer, R. A. Britton, and J. J. Tabor, "Engineering bacterial thiosulfate and tetrathionate sensors for detecting gut inflammation," Mol. Syst. Biol., vol. 13, no. 4, p. 923, 2017.

[16] C. J. McClune, A. Alvarez-Buylla, C. A. Voigt, and M. T. Laub, "Engineering orthogonal signalling pathways reveals the sparse occupancy of sequence space," Nature, vol. 574, no. 7780, pp. 702-706, 2019.

[17] J. Keener and M. Nomura, "Regulation of Ribosome Synthesis," Escherichia coli Salmonella Cell. Mol. Biol., pp. 1417-1431, 1996.

[18] B. J. Paul, W. Ross, T. Gaal, and R. L. Gourse, "rRNA transcription in Escherichia coli," Annu. Rev. Genet., vol. 38, pp. 749-770, 2004.

[19] K. Potrykus and M. Cashel, "Growth at best and worst of times," Nat. Microbiol., vol. 3, no. 8, pp. 862-863, 2018.

[20] R. L. Gourse, T. Gaal, M. S. Bartlett, J. A. Appleman, and W. Ross, "rRNA transcription and growth ratedependent regulation of ribosome synthesis in Escherichia coli.," Annu. Rev. Microbiol., vol. 50, pp. 645-77, oct 1996.

[21] F. C. Neidhardt, "Bacterial growth: Constant obsession with dN/dt," J. Bacteriol., vol. 181, no. 24, pp. 74057408, 1999. 
[22] P. P. Dennis and H. Bremer, "Modulation of Chemical Composition and Other Parameters of the Cell at Different Exponential Growth Rates," EcoSal Plus, vol. 3, no. 1, 2008.

[23] M. Scott, E. M. Mateescu, Z. Zhang, and T. Hwa, "Interdependence of Cell Growth Origins and Consequences," Science (80-. )., vol. 330, no. November, pp. 1099-1102, 2010.

[24] E. Bosdriesz, D. Molenaar, B. Teusink, and F. J. Bruggeman, "How fast-growing bacteria robustly tune their ribosome concentration to approximate growth-rate maximization," FEBS J., vol. 282, no. 10, pp. 2029-2044, 2015 .

[25] P. P. Dennis, M. Ehrenberg, and H. Bremer, "Control of rRNA synthesis in Escherichia coli: a systems biology approach.," Microbiol Mol Biol Rev, vol. 68, no. 4, pp. 639-668, 2004.

[26] J. M. Zengel and L. Lindahl, "Diverse Mechanisms for Regulating Ribosomal Protein Synthesis in Escherichia coli," Prog. Nucleic Acid Res. Mol. Biol., 1994.

[27] M. Schaechter, O. MaalOe, and N. O. Kjeldgaard, "Dependency on Medium and Temperature of Cell Size and Chemical Composition during Balanced Growth of Salmonella typhimurium," J. Gen. Microbiol., 1958.

[28] J. A. Appleman, W. Ross, J. Salomon, and R. L. Gourse, "Activation of Escherichia coli rRNA transcription by FIS during a growth cycle," J. Bacteriol., 1998.

[29] O. Schröder and R. Wagner, "The bacterial DNA-binding protein H-NS represses ribosomal RNA transcription by trapping RNA polymerase in the initiation complex," J. Mol. Biol., 2000.

[30] T. Gaal, M. S. Bartlett, W. Ross, C. L. Turnbough, and R. L. Gourse, "Transcription regulation by initiating NTP concentration: rRNA synthesis in bacteria," Science (80-. )., 1997.

[31] D. A. Schneider, T. Gaal, and R. L. Gourse, "NTP-sensing by rRNA promoters in Escherichia coli is direct," Proc. Natl. Acad. Sci. U. S. A., 2002.

[32] M. Cashel and J. Gallant, "Two compounds implicated in the function of the RC gene of escherichia coli," Nature, 1969.

[33] D. A. Schneider and R. L. Gourse, "Changes in the concentrations of guanosine 5-diphosphate 3-diphosphate and the initiating nucleoside triphosphate account for inhibition of rRNA transcription in fructose-1,6-diphosphate aldolase (fda) mutants," J. Bacteriol., 2003.

[34] K. Potrykus, H. Murphy, N. Philippe, and M. Cashel, "ppGpp is the major source of growth rate control in E. coli," Environ. Microbiol., vol. 13, no. 3, pp. 563-575, 2011.

[35] N. C. Imholz, M. J. Noga, N. J. van den Broek, and G. Bokinsky, "Calibrating the Bacterial Growth Rate Speedometer: A Re-evaluation of the Relationship Between Basal ppGpp, Growth, and RNA Synthesis in Escherichia coli," Front. Microbiol., vol. 11, no. September, pp. 1-9, 2020.

[36] E. Sarubbi, K. E. Rudd, and M. Cashel, "Basal ppGpp level adjustment shown by new spoT mutants affect steady state growth rates and rrnA ribosomal promoter regulation in Escherichia coli," MGG Mol. Gen. Genet., vol. 213, no. 2-3, pp. 214-222, 1988.

[37] M. Zacharias, H. U. Goringer, and R. Wagner, "Influence of the GCGC discriminator motif introduced into the ribosomal RNA P2- and tac promoter on growth-rate control and stringent sensitivity," EMBO J., vol. 8, no. 11, pp. 3357-3363, 1989.

[38] P. P. Dennis and H. Bremer, "Modulation of Chemical Composition and Other Parameters of the Cell at Different Exponential Growth Rates," EcoSal Plus, vol. 3, no. 1, 2008.

[39] X. Dai and M. Zhu, "Coupling of Ribosome Synthesis and Translational Capacity with Cell Growth," Trends Biochem. Sci., vol. 45, pp. 681-692, aug 2020.

[40] G. C. Atkinson, T. Tenson, and V. Hauryliuk, "The RelA/SpoT Homolog (RSH) superfamily: Distribution and functional evolution of ppgpp synthetases and hydrolases across the tree of life," PLoS One, vol. 6, no. 8, 2011.

[41] V. Hauryliuk, G. C. Atkinson, K. S. Murakami, T. Tenson, and K. Gerdes, "Recent functional insights into the role of (p)ppGpp in bacterial physiology," Nat. Rev. Microbiol., vol. 13, no. 5, pp. 298-309, 2015.

[42] L. Fernández-Coll and M. Cashel, "Possible Roles for Basal Levels of (p)ppGpp: Growth Efficiency Vs. Surviving Stress," Front. Microbiol., vol. 11, no. October, 2020.

[43] K. D. Murray and H. Bremer, "Control of spoT-dependent ppGpp Synthesis and Degradation in Escherichia coli," J. Mol. Biol, vol. 259, pp. 41-57, 1996.

[44] J. Ryals, R. Little, and H. Bremer, "Control of rRNA and tRNA syntheses in Escherichia coli by guanosine tetraphosphate," J. Bacteriol., vol. 151, no. 3, pp. 1261-1268, 1982.

[45] V. J. Hernandez and H. Bremer, "Guanosine tetraphosphate (ppGpp) dependence of the growth rate control of rrnB P1 promoter activity in Escherichia coli," J. Biol. Chem., vol. 265, no. 20, pp. 11605-11614, 1990. 
[46] H. Xiao, M. Kalman, K. Ikehara, S. Zemel, G. Glaser, and M. Cashel, "Residual guanosine 3???,5???bispyrophosphate synthetic activity of relA null mutants can be eliminated by spoT null mutations," J. Biol. Chem., vol. 266, no. 9, pp. 5980-5990, 1991.

[47] D. R. Gentry and M. Cashel, "Mutational analysis of the Escherichia coli spoT gene identifies distinct but overlapping regions involved in ppGpp synthesis and degradation," Mol. Microbiol., vol. 19, no. 6, pp. 13731384, 1996.

[48] J. M. Rock, F. F. Hopkins, A. Chavez, M. Diallo, M. R. Chase, E. R. Gerrick, J. R. Pritchard, G. M. Church, E. J. Rubin, C. M. Sassetti, D. Schnappinger, and S. M. Fortune, "Programmable transcriptional repression in mycobacteria using an orthogonal CRISPR interference platform," Nat. Microbiol., vol. 2, no. February, pp. 1-9, 2017.

[49] Y. J. Lee, A. Hoynes-O'Connor, M. C. Leong, and T. S. Moon, "Programmable control of bacterial gene expression with the combined CRISPR and antisense RNA system," Nucleic Acids Res., vol. 44, no. 5, pp. 2462-2473, 2016.

[50] H. H. Huang, M. Bellato, Y. Qian, P. Cárdenas, L. Pasotti, P. Magni, and D. Del Vecchio, "dCas9 regulator to neutralize competition in CRISPRi circuits," Nat. Commun., vol. 12, no. 1, pp. 1-7, 2021.

[51] F. Ceroni, R. Algar, G.-B. Stan, and T. Ellis, "Quantifying cellular capacity identifies gene expression designs with reduced burden," Nat. Methods, vol. 12, no. 5, pp. 415-418, 2015.

[52] T. Durfee, R. Nelson, S. Baldwin, G. Plunkett, V. Burland, B. Mau, J. F. Petrosino, X. Qin, D. M. Muzny, M. Ayele, R. A. Gibbs, B. Cs??rgo, G. P??sfai, G. M. Weinstock, and F. R. Blattner, "The complete genome sequence of Escherichia coli DH10B: Insights into the biology of a laboratory workhorse," J. Bacteriol., vol. 190, no. 7, pp. 2597-2606, 2008.

[53] K. Valgepea, K. Adamberg, A. Seiman, and R. Vilu, "Escherichia coli achieves faster growth by increasing catalytic and translation rates of proteins," Mol. Biosyst., vol. 9, no. 9, p. 2344, 2013.

[54] M. Zhu, Y. Pan, and X. Dai, “(p)ppGpp: the magic governor of bacterial growth economy," Curr. Genet., vol. 65, no. 5, pp. 1121-1125, 2019.

[55] L. U. Magnusson, A. Farewell, and T. Nyström, "ppGpp: A global regulator in Escherichia coli," Trends Microbiol., vol. 13, no. 5, pp. 236-242, 2005.

[56] V. J. Hernandez and H. Bremer, "Characterization of RNA and DNA synthesis in Escherichia coli strains devoid of ppGpp," J. Biol. Chem., vol. 268, no. 15, pp. 10851-10862, 1993.

[57] M. Zhu, H. Mu, M. Jia, L. Deng, and X. Dai, "Control of ribosome synthesis in bacteria: the important role of rRNA chain elongation rate," Sci. China Life Sci., vol. 3, pp. 169-84, aug 2020.

[58] T. H. Segall-Shapiro, E. D. Sontag, and C. A. Voigt, "Engineered promoters enable constant gene expression at any copy number in bacteria," Nat. Biotechnol., vol. 36, no. 4, pp. 352-358, 2018.

[59] E. Dekel and U. Alon, "Optimality and evolutionary tuning of the expression level of a protein," Nature, vol. 436, no. 7050, pp. 588-592, 2005.

[60] M. C. Chan, S. Karasawa, H. Mizuno, I. Bosanac, D. Ho, G. G. Privé, A. Miyawaki, and M. Ikura, "Structural characterization of a blue chromoprotein and its yellow mutant from the sea anemone Cnidopus japonicus," $J$. Biol. Chem., vol. 281, no. 49, pp. 37813-37819, 2006.

[61] P. D. Hsu, D. A. Scott, J. A. Weinstein, F. A. Ran, S. Konermann, V. Agarwala, Y. Li, E. J. Fine, X. Wu, O. Shalem, T. J. Cradick, L. A. Marraffini, G. Bao, and F. Zhang, "DNA targeting specificity of RNA-guided Cas9 nucleases," Nat. Biotechnol., vol. 31, no. 9, pp. 827-832, 2013.

[62] S. H. Sternberg, S. Redding, M. Jinek, E. C. Greene, and J. A. Doudna, "DNA interrogation by the CRISPR RNA-guided endonuclease Cas9," Nature, vol. 507, no. 7490, pp. 62-67, 2014.

[63] D. Del Vecchio and R. M. Murray, Biomolecular Feedback Systems. 2014.

[64] D. Sun, G. Lee, J. H. Lee, H. Y. Kim, H. W. Rhee, S. Y. Park, K. J. Kim, Y. Kim, B. Y. Kim, J. I. Hong, C. Park, H. E. Choy, J. H. Kim, Y. H. Jeon, and J. Chung, "A metazoan ortholog of SpoT hydrolyzes ppGpp and functions in starvation responses," Nat. Struct. Mol. Biol., vol. 17, no. 10, pp. 1188-1194, 2010.

[65] M. Zhu and X. Dai, "Growth suppression by altered (p)ppGpp levels results from non-optimal resource allocation in Escherichia coli," Nucleic Acids Res., vol. 47, no. 9, pp. 4684-4693, 2019.

[66] R. Harinarayanan, H. Murphy, and M. Cashel, "Synthetic growth phenotypes of Escherichia coli lacking ppGpp and transketolase A ( tktA ) are due to ppGpp-mediated transcriptional regulation of tktB," Mol. Microbiol., vol. 69, pp. 882-894, aug 2008.

[67] A. P. S. Darlington, J. Kim, J. I. Jiménez, and D. G. Bates, "Dynamic allocation of orthogonal ribosomes facilitates uncoupling of co-expressed genes," Nat. Commun., vol. 9, no. 1, 2018. 
[68] S. K. Aoki, G. Lillacci, A. Gupta, A. Baumschlager, D. Schweingruber, and M. Khammash, "A universal biomolecular integral feedback controller for robust perfect adaptation," Nature, vol. 570, no. 7762, pp. 533-537, 2019.

[69] H. H. Huang, Y. Qian, and D. Del Vecchio, "A quasi-integral controller for adaptation of genetic modules to variable ribosome demand," Nat. Commun., vol. 9, no. 1, pp. 1-12, 2018.

[70] D. G. Gibson, L. Young, R. Y. Chuang, J. C. Venter, C. A. Hutchison, and H. O. Smith, "Enzymatic assembly of DNA molecules up to several hundred kilobases," Nat. Methods, vol. 6, no. 5, pp. 343-345, 2009.

[71] D. Del Vecchio, R. M. Murray, and D. Vecchio, Biomolecular Feedback Systems. 2010.

[72] A. G. Marr, "Growth Rate of Escherichia coli," Microbiol. Mol. Biol. Rev., vol. 55, no. 2, pp. 316-333, 1991.

[73] H. D. Murray, J. A. Appleman, and R. L. Gourse, "Regulation of the Escherichia coli rrnB P2 promoter," J. Bacteriol., vol. 185, no. 1, pp. 28-34, 2003.

[74] M. M. Barker, T. Gaal, C. A. Josaitis, and R. L. Gourse, "Mechanism of regulation of transcription initiation by ppGpp. I. Effects of ppGpp on transcription initiation in vivo and in vitro," J. Mol. Biol., vol. 305, no. 4, pp. 673-688, 2001.

[75] Y. Qian and D. Del Vecchio, "Mitigation of ribosome competition through distributed sRNA feedback," 2016 IEEE 55th Conf. Decis. Control. CDC 2016, pp. 758-763, 2016.

[76] H. M. Salis, E. A. Mirsky, and C. A. Voigt, "Automated design of synthetic ribosome binding sites to control protein expression," Nat. Biotechnol., 2009.

[77] A. Espah Borujeni, A. S. Channarasappa, and H. M. Salis, "Translation rate is controlled by coupled trade-offs between site accessibility, selective RNA unfolding and sliding at upstream standby sites," Nucleic Acids Res., vol. 42, no. 4, pp. 2646-2659, 2014.

\section{Supplementary Information}

In the first section of the SI we provide supplementary experimental results. Then, we provide a detailed mathematical derivation of the SpoTH actuator model. Finally, we provide the plasmid maps of the constructs used in this study along with DNA sequence of nonstandard parts.

\section{Additional experimental data}

The actuation profile when expressing SpoTH in the DH10B strain is shown in Fig. 10. The unnormalzied GFP production rate and growth rate corresponding to Fig. 2-d and Fig. 2-f in the main text, respectively, are shown in Fig. 11. The GFP production rate and growth rate when carrying out the experiments corresponding to Fig. 3 in the main text, but in the wild-type MG1655 strain is shown in Fig. 12. The RFP production rate, the unnormalized GFP production rate, and the unnormarlized growth rate as AHL induction increases for the open loop and closed loop data corresponding to Fig. 4-f and Fig. 4-g in the main text, is shown Fig. 13. The RFP production rate, the unnormalized GFP production rate, and the unnormarlized growth rate as AHL induction increases for the control data corresponding to Fig. 4-f and Fig. 4-g in the main text, is shown Fig. 14. The RFP production rate, the unnormalized GFP production rate, and the unnormarlized growth rate as AHL induction increases for the open loop and closed loop data corresponding to Fig. 4-h and Fig. 4-i in the main text, is shown Fig. 15. The RFP production rate, the unnormalized GFP production rate, and the unnormarlized growth rate as AHL induction increases for the control data corresponding to Fig. 4-h and Fig. 4-i in the main text, is shown Fig. 16. The unnormarlized data corresponding to Fig. 5 in the main text, is shown in Fig. 17.

\section{Derivation of the SpoTH actuator mathematical model}

Following the deterministic modeling framework in [71] and previously applied in [1, 6], we derive a model of the SpoTH actuator. We model SpoTH mRNA being translated by ribosomes to produce the SpoTH protein, which catalyzes the hydrolysis of ppGpp. We model ppGpp inhibiting ribosome production and thus modifying the total ribosomal budget. The resulting dimensional model contains many free parameters, by nondimensionalizing the equations, we can reduce our governing equation to contain only two dimensionless parameters. Finally, we modify the equations to account for the expression of a heterologous protein.

This modeling framework is not meant to be comprehensive, but rather contain sufficient fidelity to make mathematically precise the physical processes discussed in the main text. The mathematical model is meant to complement the physical intuition provided in the main text used to explain the experimental data. 
bioRxiv preprint doi: https://doi.org/10.1101/2021.02.11.430724; this version posted June 24, 2021. The copyright holder for this preprint (which was not certified by peer review) is the author/funder, who has granted bioRxiv a license to display the preprint in perpetuity. It is made available under aCC-BY-NC-ND 4.0 International license.

a

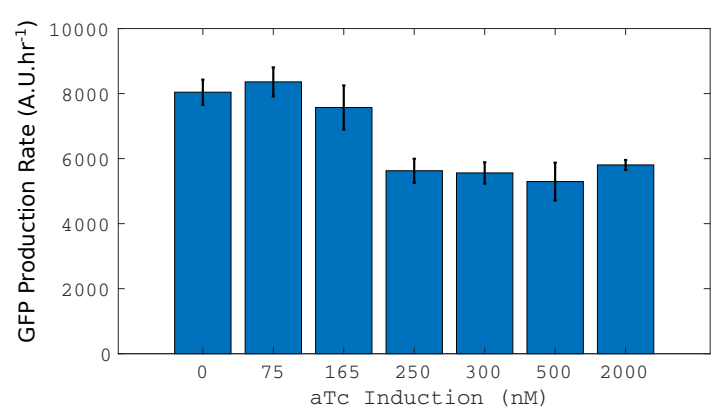

b

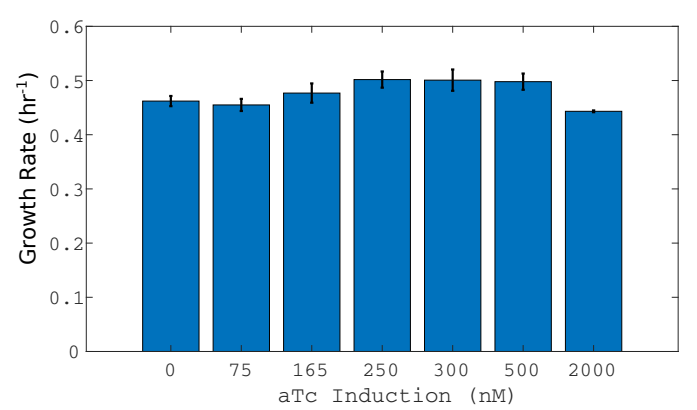

Figure 10: The actuation profile in DH10B (a)/ (b) The GFP production rate/growth rate when expressing SpoTH using the genetic construct shown in Fig. 2-a in the main text in the DH10B strain. Other than the aTc induction levels, all experimental conditions are identical to those used to generate Fig. 2-c/Fig. 2-d in the main text.

a

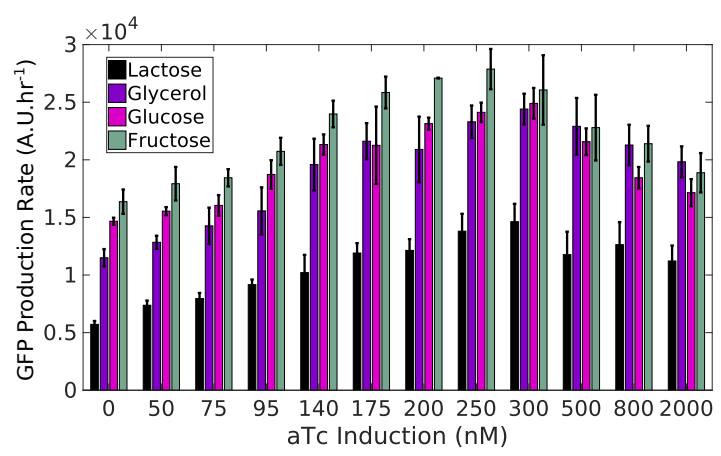

b

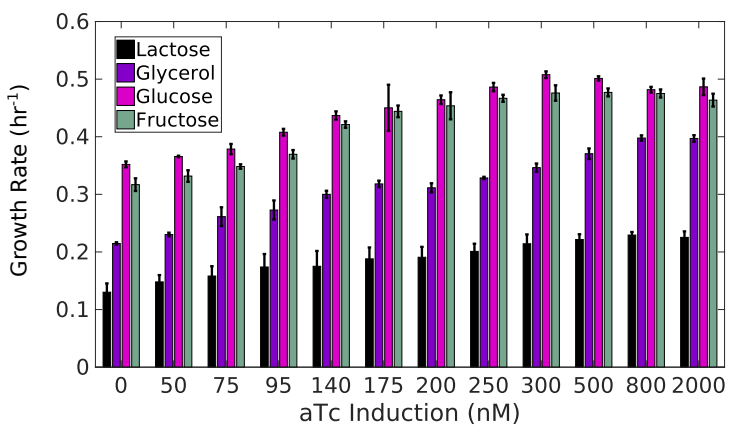

Figure 11: The unnormalized data corresponding to Fig. 2 in the main text (a) The unnormalized GFP production rate corresponding to Fig. 2-e in the main text (b) The unnormalzied growth rate corresponding to Fig. 2-g in the main text.

a

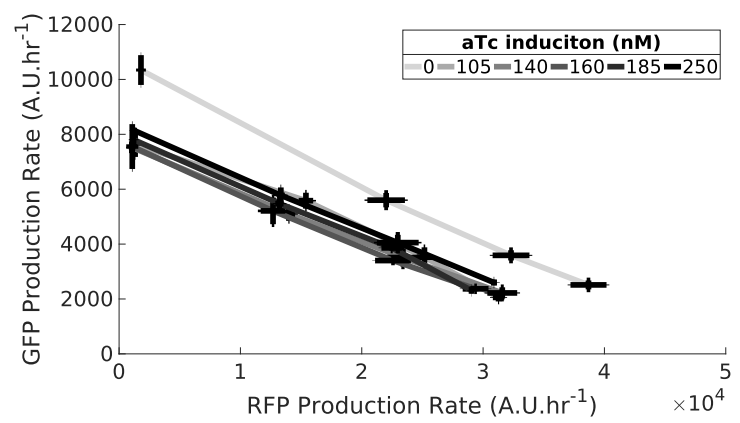

b

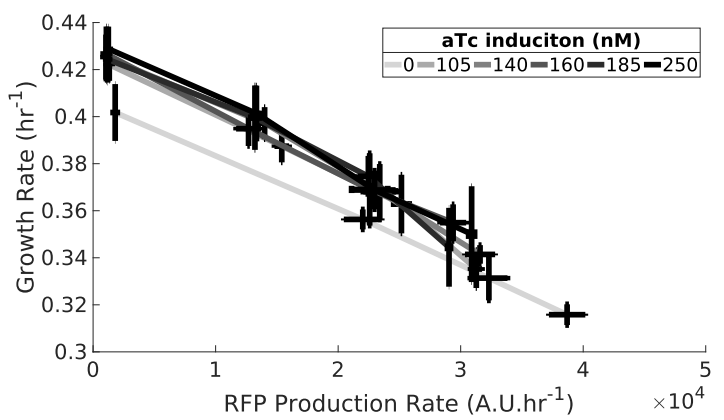

Figure 12: Simultaneously expressing SpoTH and RFP in the wild-type MG1655 strain. The P_GFP_SpoTH_RFP plasmid is used to express SpoTH using the inducible TetR-pTet promoter and RFP using the inducible luxR-Plux. Plasmid description, plasmid map, and essential DNA sequences are provided in SI section Plasmid maps and DNA sequences. (a) The GFP production rate as RFP production rate is increased, for different levels of SpoTH induction (denoted by shades of gray). (b) The cell growth rate as RFP production rate is increased, for different levels of SpoTH induction (denoted by shades of gray). Data are shown as the mean \pm one standard deviation $(\mathrm{N}=4$, two biological replicates each with two technical replicates). All experiments were performed in the MG1655 strain in media with glycerol as the sole carbon source. The AHL concentrations used in this experiment were AHL $=0 \mathrm{nM}, 3 \mathrm{nM}, 7 \mathrm{nM}, 15 \mathrm{nM}$. The complete experimental protocol is provided in the Materials and Methods section.

\section{SpoTH expression and ppGpp hydrolysis and synthesis}

We model SpoTH mRNA $\left(\mathrm{m}_{\mathrm{s}}\right)$ binding to free ribosomes $(\mathrm{R})$ to produce the translation initiation complex $\mathrm{c}_{\mathrm{s}}$, which is then translated to produce the SpoTH protein $\mathrm{S}$ with elongation rate constant $\kappa_{s}$. The mRNA decays with rate 

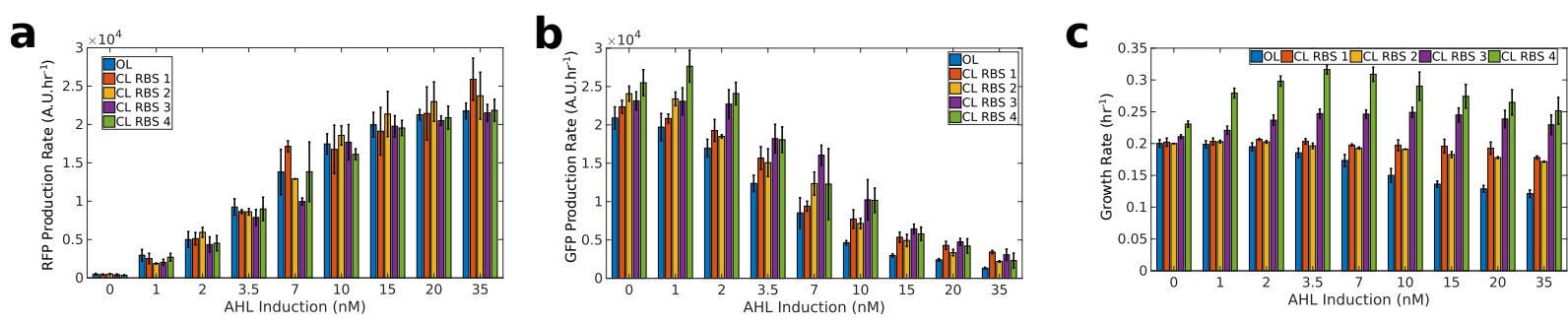

Figure 13: The unnormalized open loop and closed loop data corresponding to the glycerol experiment in Fig. 4 in the main text. (a) The RFP production rate vs AHL induction data corresponding to Fig. 2-h in the main text for the open loop and closed loop experiments. (b) The unnormalized GFP production rate vs AHL induction data corresponding to Fig. 2-h in the main text for the open loop and closed loop experiments. (c) The unnormalized growth rate vs AHL induction data corresponding to Fig. 2-i in the main text for the open loop and closed loop experiments.
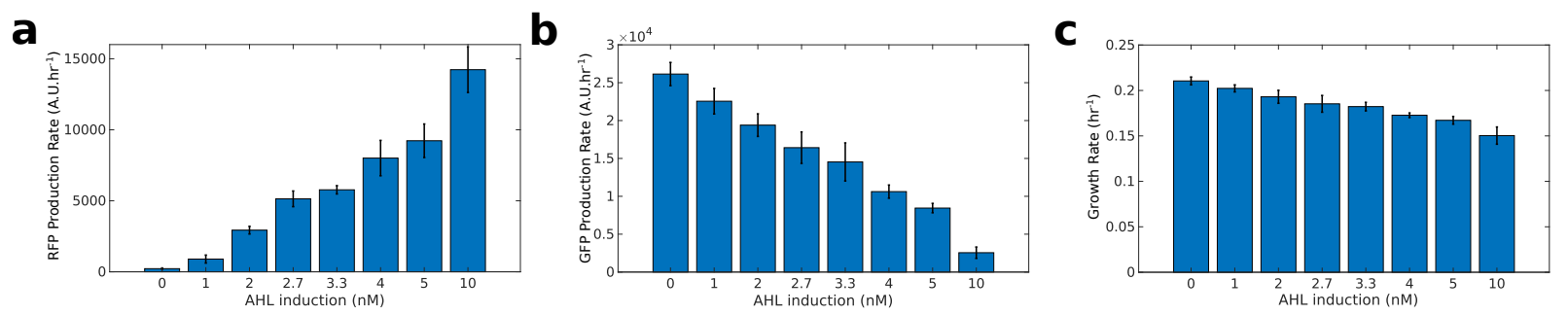

Figure 14: The unnormalized control data corresponding to the glycerol experiment in Fig. 4 in the main text. (a) The RFP production rate vs AHL induction data corresponding to Fig. 2-h in the main text for the control experiment. (b) The unnormalized GFP production rate vs AHL induction data corresponding to Fig. 2-h in the main text for the control experiment. (c) The unnormalized growth rate vs AHL induction data corresponding to Fig. 2-i in the main text for the control experiment.
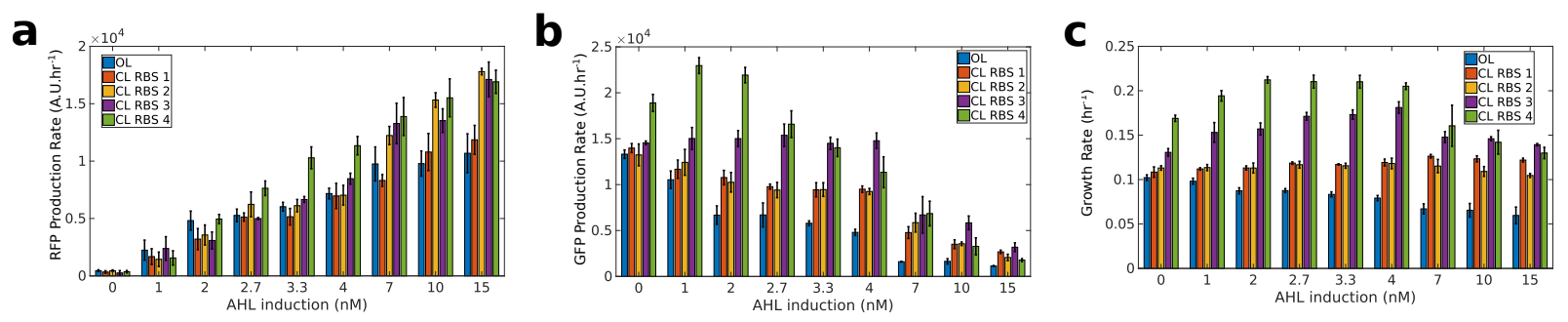

Figure 15: The unnormalized open loop and closed loop data corresponding to the lactose experiment in Fig. 4 in the main text. (a) The RFP production rate vs AHL induction data corresponding to Fig. 2-f in the main text for the open loop and closed loop experiments. (b) The unnormalized GFP production rate vs AHL induction data corresponding to Fig. 2-f in the main text for the open loop and closed loop experiments. (c) The unnormalized growth rate vs AHL induction data corresponding to Fig. 2-g in the main text for the open loop and closed loop experiments.

constants $\delta_{s}$ and the protein dilutes with rate constant $\gamma_{s}$. The corresponding chemical reactions are:

$$
\emptyset \stackrel{\alpha_{\mathrm{s}}}{\longrightarrow} \mathrm{m}_{\mathrm{s}}, \quad \mathrm{m}_{\mathrm{s}} \stackrel{\delta_{\mathrm{s}}}{\longrightarrow} \emptyset, \quad \mathrm{m}_{\mathrm{s}}+\mathrm{R} \underset{\mathrm{d}_{\mathrm{s}}}{\stackrel{\mathrm{a}_{\mathrm{s}}}{\rightleftharpoons}} \mathrm{c}_{\mathrm{s}} \stackrel{\kappa_{\mathrm{s}}}{\longrightarrow} \mathrm{m}_{\mathrm{s}}+\mathrm{R}+\mathrm{P}_{\mathrm{s}}, \quad \mathrm{S} \stackrel{\gamma_{\mathrm{s}}}{\longrightarrow} \emptyset
$$

where $\alpha_{s}$ is the production rate constant of the mRNA, $a_{s}$ and $d_{s}$ are the association and dissociation rate constant, respectively, between ribosomes and mRNA. Levering reaction rate equations, consequently, the concentration of each 
a

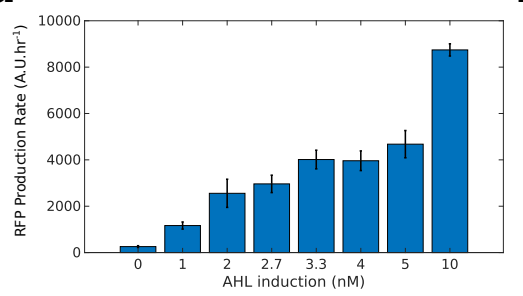

b

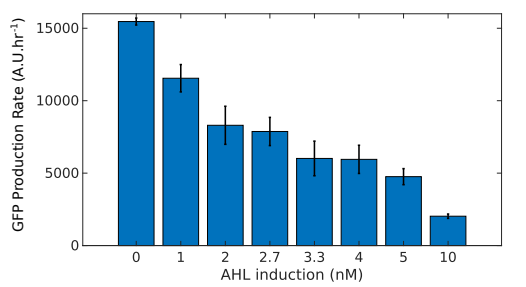

C

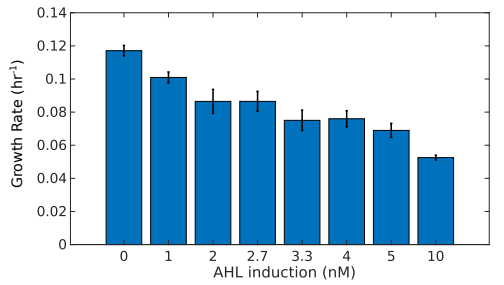

Figure 16: The unnormalized control data corresponding to the lactose experiment in Fig. 4 in the main text. (a) The RFP production rate vs AHL induction data corresponding to Fig. 2-f in the main text for the control experiment. (b) The unnormalized GFP production rate vs AHL induction data corresponding to Fig. 2-f in the main text for the control experiment. (c) The unnormalized growth rate vs AHL induction data corresponding to Fig. 2-g in the main text for the control experiment.

a

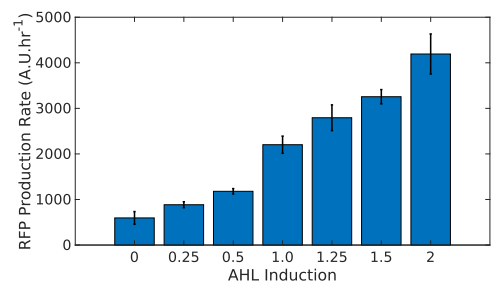

b

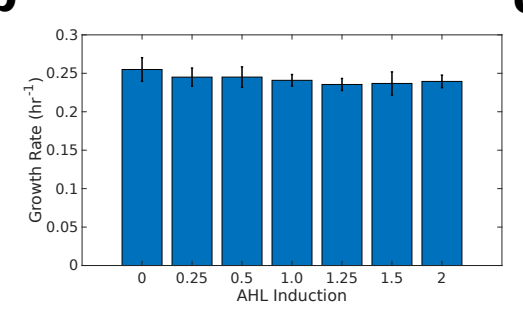

C

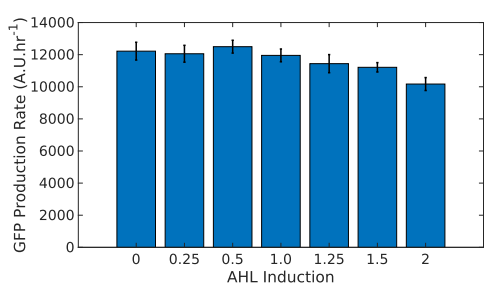

d

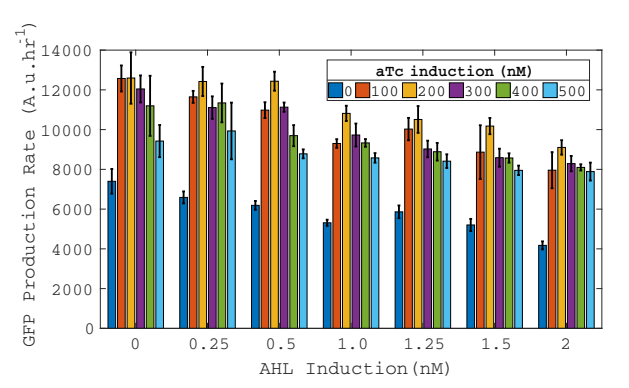

Figure 17: Supplemental data to Fig. 5 in the main text (a) The RFP production rate from inducing P_GFP_SpoTH_RFP using the same AHL induction levels as those from Fig. 5-b in main text and no aTc induction. (b) The growth rate from inducing $\mathrm{P} \_$GFP_SpoTH_RFP using the same AHL induction levels as those from Fig. 5-b in main text and no aTc induction. (c) The GFP production rate from inducing P_GFP_SpoTH_RFP using the same AHL induction levels as those from Fig. 5-b in main text and no aTc induction. (d) The GFP production rate from inducing P_GFP_SpoTH_dCas9 using the same AHL and aTC induction levels as those from Fig. 5-b in main text.

species satisfies:

$$
\begin{aligned}
\frac{d m_{s}}{d t} & =\alpha_{s}-a_{s} R m_{s}+\left(d_{s}+\kappa_{s}\right) c_{s}-\delta_{s} m_{s} \\
\frac{d c_{s}}{d t} & =a_{s} R m_{s}-\left(d_{s}+\kappa_{s}\right) c_{s} \\
\frac{d S}{d t} & =\kappa_{s} c_{s}-\gamma_{s} S .
\end{aligned}
$$

The steady state of (10) is given by

$$
m_{s}=\frac{\alpha_{s}}{\delta_{s}}, \quad c_{s}=\frac{m_{s}}{K_{s}} R, \quad S=\frac{\kappa_{s}}{\gamma_{s}} c_{s}
$$

where $K_{s}=\frac{d_{s}+\kappa_{s}}{a_{s}}$. From (11), the concentration of SpoTH $S$ is proportional to $c_{s}$ (the number of ribosomes translating SpoTH mRNA). 
We model RelA (A) and endogenous SpoT $\left(\mathrm{S}_{0}\right)$ catalyzing the synthesis of ppGpp $(\mathrm{G})$ (as in Fig. 1 in the main text) from GTP/GDP $\left(\mathrm{G}_{\mathrm{P}}\right)$, with rates $s_{1}$ and $s_{2}$, respectively. We model endogenous SpoT and SpoTH catalyzing the hydrolysis of ppGpp to GTP/GDP $\left(\mathrm{G}_{\mathrm{P}}\right)$ with rates $h_{1}$ and $h_{2}$, respectively. For simplicity, we model these processes using a one-step reaction model [71], that is,

$$
\mathrm{A}+\mathrm{G}_{\mathrm{P}} \stackrel{\mathrm{s}_{1}}{\longrightarrow} \mathrm{G}+\mathrm{A}, \quad \mathrm{S}_{0}+\mathrm{G}_{\mathrm{P}} \stackrel{\mathrm{s}_{2}}{\longrightarrow} \mathrm{G}+\mathrm{S}_{0}, \quad \mathrm{~S}_{0}+\mathrm{G} \stackrel{\mathrm{h}_{1}}{\longrightarrow} \mathrm{G}_{\mathrm{P}}+\mathrm{S}_{0}, \quad \mathrm{~S}+\mathrm{G} \stackrel{\mathrm{h}_{2}}{\longrightarrow} \mathrm{G}_{\mathrm{P}}+\mathrm{S} .
$$

The concentration of ppGpp satisfies:

$$
\frac{d G}{d t}=\alpha_{G}-\gamma_{G_{0}} G-h_{2} G S
$$

where $\alpha_{G}=s_{1} A G_{P}+s_{2} S_{0} G_{P}$ is the effective production rate and $\gamma_{G_{0}}=h_{1} S_{0}$ is the basal decay rate. The steady state of (13) is given by

$$
G=\frac{G_{0}}{1+S / K_{g s}}
$$

where $K_{g s}=\gamma_{G_{0}} / h_{2}$ and $G_{0}=\alpha_{G} / \gamma_{G_{0}}$. The quantity $G_{0}$ corresponds to the basal ppGpp in the cell $\left(G(S=0)=G_{0}\right)$.

\section{Actuating the ribosomal budget in the cell}

The concentration of total ribosomes in the cell $\left(R_{T}\right)$, known as the ribosomal budget [1], is composed of free ribosomes, the portion of ribosomes translating endogenous mRNAs $\left(c_{e}\right)$, and the portion of ribosomes translating SpoTH mRNA, that is,

$$
R_{T}=R+c_{s}+c_{e}
$$

The total ribosome concentration obeys

$$
\frac{d R_{T}}{d t}=\alpha_{r}-\gamma_{r} R_{T}
$$

where $\alpha_{r}$ is the ribosome production rate, $\gamma_{r}$ is the ribosome decay rate. If $\alpha_{r}$ and $\gamma_{r}$ are assumed time invariant and $R_{T}(0)=\frac{\alpha_{r}}{\gamma_{r}}$, then $R_{T}(t)=\frac{\alpha_{r}}{\gamma_{r}}, \forall t \geq 0$. A temporally constant ribosome budget is consistent with the modeling framework of $[1,6,69]$. However, in this work $\alpha_{r}$ is not constant and is the term that links ribosome and ppGpp concentration.

Ribosome production $\left(\alpha_{r}\right)$ is set by rRNA production (this the rate-limiting step) [18, 19]. rRNA is expressed from seven rRNA operons ( $r r n$ operons) [72] each driven by two tandem promoters P1 and P2. Most rRNA transcription arises from the P1 promoter and it is the main "knob" for ribosome tuning except at very low growth rates where P2 regulation dominates [73]. During balanced exponential growth, ppGpp is the primary regulator of rRNA[18, 34, 35] by destabilizing the open RNAP-P1 promoter complex [74, 25]. Therefore, there is an inverse relationship between basal ppGpp levels and rRNA transcription [36, 37, 38, 39]. A simple model to capture this process (previously used in [3]), is given by

$$
\alpha_{r}=\frac{\alpha_{r}^{*}}{1+\left(G / K_{G}\right)^{2}}
$$

where $K_{G}$ is the effective dissociation constant between ppGpp and the P1 promoter and $\alpha_{r}^{*}$ is the ribosome production rate in the absence of ppGpp $\left(\alpha_{r}(G=0)\right)$. The hill coefficient of 2 in (17) is consistent with the findings of [72]. Taking the steady state of (16) and levering (11), (14), and (17), we have that

$$
R_{T}=\frac{R_{T}^{*}}{1+\left(G / K_{G}\right)^{2}}=\frac{R_{T}^{*}}{1+\left(\frac{G_{0}}{K_{G}}\left(\frac{1}{1+c_{s} \frac{\kappa_{s}}{K_{g s} \gamma_{s}}}\right)\right)^{2}},
$$

where $R_{T}^{*}=\alpha_{r}^{*} / \gamma_{r}$.

Rewriting (15), we have at steady state that

$$
\frac{R_{T}^{*}}{1+\left(\frac{G_{0}}{K_{G}}\left(\frac{1}{1+c_{s} \frac{\kappa_{s}}{K_{g s \gamma_{s}}}}\right)\right)^{2}}=R+c_{s}+c_{e}
$$

making explicit the relationship between basal ppGpp concentration $\left(G_{0}\right)$ and the total ribosomal budget and how increasing SpoTH expression (increasing $c_{s}$ ) both increases the total ribosomal budget (LHS) but also sequesters ribosomes (RHS) via translation demand. as

By adding and subtracting $\frac{1}{1+\left(G_{0} / K_{G}\right)^{2}}$ to the LHS of (15) and dividing both sides by $R_{T}^{*}$, we can rewrite of (15)

$$
\frac{1}{1+\left(G_{0} / K_{G}\right)^{2}}+\left[\frac{1}{1+\left(\frac{G_{0}}{K_{G}}\left(\frac{1}{1+c_{s} \frac{\kappa_{s}}{K_{g s \gamma_{s}}}}\right)\right)^{2}}-\frac{1}{1+\left(G_{0} / K_{G}\right)^{2}}\right]=\frac{R+c_{e}}{R_{T}^{*}}+\frac{c_{s}}{R_{T}^{*}}
$$

Modeling $c_{e}$ requires knowing the concentration of the mRNA-ribosome complex for every mRNA expressed by an endogenous gene and thus it is difficult to write an explicit expression. Instead of modeling $c_{e}$ explicitly, we keep it as a general function of $R$. We assume that the concentration of $c_{e}(R)$ monotonically increases with free ribosomes, 
that is, $\frac{d c_{e}}{d R}>0$. This assumptions is reasonable since a steady state complex concentration is proportional to the concentrations of the reacting species [63] (also (11)). Next, we define the following variable that serves as a proxy for free ribosomes:

$$
z(R)=R+c_{e}(R) .
$$

From the assumption that $\frac{d c_{e}}{d R}>0$, it implies that the map $z(R)$ is one-one and thus for every value of $R$ there is a unique corresponding value of $z$ and that an increase/decrease in $R$ corresponds to an increase/decrease in $z$. Furthermore, we have that $z(0)=0$ since no the complex $c_{e}$ cannot be formed without the reactant species $R$. Therefore, from here on, we refer to $z(R)$ as the modified free ribosome concentration.

Example: if we assume that $c_{e}(R)$ had a form similar to that as $c_{s}$ as given by (11), then, for $q$ different endogenous genes expressing mRNA, $c_{e}(R)=\sum_{i=1}^{q} \frac{m_{e, i}}{K_{e, i}} R$, where for gene $i, m_{e, i}$ is the endogenous mRNA concentration and $K_{e, i}$ is the effective dissociation constant of endogenous mRNA with ribosomes. In this cases, $c_{e}(R)$ satisfies all of our assumptions and furthermore, $z(R)$ is simply proportional to $R$. However, in all of our analysis we do not explicitly specify $c_{e}(R)$.

We denote $\bar{z}=\frac{z}{R_{T}^{*}}$ and $\bar{c}_{s}=\frac{c_{s}}{R_{T}^{*}}$ and express (19) in dimensionless form as

$$
\bar{z}_{0}+\left(1-\bar{z}_{0}\right) f\left(\bar{c}_{s} / \epsilon, \bar{z}_{0}\right)=\bar{z}+\bar{c}_{s}
$$

where

$$
\theta_{G}=G_{0} / K_{G}, \quad \bar{z}_{0}\left(\theta_{G}\right)=\frac{1}{1+\theta_{G}^{2}}, \quad \epsilon=\frac{K_{g s}}{R_{T}^{*}} \frac{\gamma_{s}}{\kappa_{s}}, \quad f\left(\bar{c}_{s} / \epsilon, \bar{z}_{0}\right)=\frac{\left(\bar{c}_{s} / \epsilon+1\right)^{2}-1}{\left(\bar{c}_{s} / \epsilon+1\right)^{2}-1+1 / \bar{z}_{0}} .
$$

The dimensionless parameter $\theta_{G}$ is a measure of the basal ppGpp in the cell, $\bar{z}_{0}$ is the dimensionless modified free ribosome concentration when no SpoTH is expressed $\left(\bar{c}_{s}=0\right)$ and we refer to this quantity as the nominal modified ribosome level, $\epsilon$ is a measure of the ribosomal cost to express sufficient SpoTH to actuate (catalyze the hydrolysis of a sufficient amount of ppGpp). A small $\epsilon$ implies that a small $\bar{c}_{s}$ is needed to saturate the $f$ term. Also notice that there is a monotonically decreasing relationship between the basal ppGpp $\theta_{G}$ and the nominal modified ribosome level. Finally, a key parameter to determine the qualitative behavior of (20) is given by:

$$
\delta\left(\bar{z}_{0}, \epsilon\right):=\left.\frac{d \bar{z}}{d \bar{c}_{s}}\right|_{\bar{c}_{s}=0}=2 \frac{\theta_{G}^{2}}{\left(1+\theta_{G}^{2}\right)^{2} \epsilon}-1=\frac{2 \bar{z}_{0}\left(1-\bar{z}_{0}\right)}{\epsilon}-1
$$

where $\delta \in(-1, \infty)$. By definition and our assumption that $\frac{d c_{e}}{d R}>0$, if $\delta>0$, it implies that ribosome levels increase as a small amount of exogenous SpoT is expressed.

\section{Appending the model with the expression of an additional heterologous protein}

We model the mRNA of a heterologous protein $\left(\mathrm{m}_{\mathrm{y}}\right)$ binding to free ribosomes $(\mathrm{R})$ to produce the translation initiation complex $c_{y}$, which is then translated to produce the protein y with elongation rate constant $\kappa_{y}$. The mRNA decays with rate constants $\delta_{y}$ and the protein dilutes with rate constant $\gamma_{y}$. The corresponding chemical reactions are:

$$
\emptyset \stackrel{\alpha_{\mathrm{y}}}{\longrightarrow} \mathrm{m}_{\mathrm{y}}, \quad \mathrm{m}_{\mathrm{y}} \stackrel{\delta_{\mathrm{y}}}{\longrightarrow} \emptyset, \quad \mathrm{m}_{\mathrm{y}}+\mathrm{R} \underset{\mathrm{d}_{\mathrm{y}}}{\stackrel{\mathrm{a}_{\mathrm{y}}}{\rightleftharpoons}} \mathrm{c}_{\mathrm{y}} \stackrel{\kappa_{\mathrm{y}}}{\longrightarrow} \mathrm{m}_{\mathrm{y}}+\mathrm{R}+\mathrm{P}_{\mathrm{y}}, \quad \mathrm{y} \stackrel{\gamma_{\mathrm{y}}}{\longrightarrow} \emptyset
$$

where $\alpha_{y}$ is the production rate constant of the mRNA, $a_{y}$ and $d_{y}$ are the association and dissociation rate constant, respectively, between ribosomes and mRNA. The concentration of each species satisfies:

$$
\begin{aligned}
\frac{d m_{y}}{d t} & =\alpha_{y}-a_{y} R m_{y}+\left(d_{y}+\kappa_{y}\right) c_{y}-\delta_{y} m_{y} \\
\frac{d c_{y}}{d t} & =a_{y} R m_{y}-\left(d_{y}+\kappa_{y}\right) c_{y} \\
\frac{d y}{d t} & =\kappa_{y} c_{y}-\gamma_{y} P_{y} .
\end{aligned}
$$

The steady state of (24) is given by

$$
m_{y}=\frac{\alpha_{y}}{\delta_{y}}, \quad c_{y}=\frac{m_{y}}{K_{y}} R, \quad y=\frac{\kappa_{y}}{\gamma_{y}} c_{y}
$$

where $K_{y}=\frac{d_{y}+\kappa_{y}}{a_{y}}$. We modify the total ribosome equation (15) to include the ribosomes sequestered by the $\mathrm{y}$ mRNA, and it reads

$$
R_{T}=R+c_{s}+c_{e}+c_{y}
$$

Defining $\bar{c}_{y}=c_{y} / R_{T}^{*}$, the total ribosome concentration in dimensionless from as in (20), is given by

$$
\bar{z}_{0}+\left(1-\bar{z}_{0}\right) f\left(\bar{c}_{s} / \epsilon, \bar{z}_{0}\right)=\bar{z}+\bar{c}_{s}+\bar{c}_{y} .
$$



state

If $\mathrm{y}$ and SpoTH are under the same promoter, that is $m_{y}=m_{s}$, then from (11) and (25) we have that at steady

$$
\bar{c}_{s}=\gamma \bar{c}_{y},
$$

where $\gamma=K_{y} / K_{s}$ is the SpoTH RBS strength relative to the y RBS strength. We refer to the configuration when SpoTH and y are under the same promoter $\left(\bar{c}_{s}=\gamma \bar{c}_{y}\right)$, as the closed loop and the case when y is expressed in isolation $\left(\bar{c}_{s}=0\right.$ for all $\left.\bar{c}_{y}\right)$, as the open loop. The qualitative behavior of (26) for the open loop and closed loop is shown in Fig. 18. For the close loop, we have can express the initial sensitivity of free ribosome as y is expressed as

$$
\left.\frac{d \bar{z}}{d \bar{c}_{y}}\right|_{\bar{c}_{y}=0}=\gamma \delta-1 .
$$

Thus, we can make the slope zero (free ribosomes are initially not sensitive to the expression of y) if we choose the SpoTH RBS strength (relative to the y RBS strength) as

$$
\gamma=1 / \delta\left(\bar{z}_{0}, \epsilon\right)
$$

In Fig. 18 we observe that as $\gamma \delta \rightarrow-1$, the closed loop performs worst than the open loop (for a given $\bar{c}_{y}$, the corresponding value of $\bar{z}$ is lower) and the performance gets worst for larger values of $\gamma$.

\section{Qualitive behavior of open loop and closed loop systems}

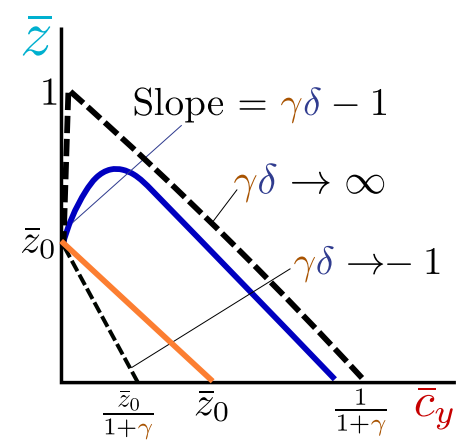

Figure 18: The qualitative behavior coexpressing y and SpoTH under the same promoter. The qualitative behavior of (26) when $\bar{c}_{s}=\gamma \bar{c}_{y}$ (blue line). The open loop $\left(\bar{c}_{s}=0\right.$ for all $\left.\bar{c}_{y}\right)$ is shown in orange. The asymptotic behaviors as $\gamma \delta \rightarrow-1$ and $\gamma \delta \rightarrow \infty$ are shown in dashed lines. 
bioRxiv preprint doi: https://doi.org/10.1101/2021.02.11.430724; this version posted June 24, 2021. The copyright holder for this preprint (which was not certified by peer review) is the author/funder, who has granted bioRxiv a license to display the preprint in perpetuity. It is made available under aCC-BY-NC-ND 4.0 International license.

\section{Plasmid maps and DNA sequences}

The plasmids used in this study and their description are provided in Table 1. The corresponding plasmid maps are shown in Fig. 21. The essential DNA sequences are provided in Table 2. The full plasmid DNA sequences are uploaded to Addgene (\#xxx-xx, will specify once study is finalized).

\begin{tabular}{|c|c|c|}
\hline Plasmid Name & Plasmid map & Comments \\
\hline P_GFP_SpoTH & Fig. 21-a & $\begin{array}{c}\text { SpoTH cloned using pHX41 [47], see Supplementary Note } 3 \\
\text { The rest of the parts are from pHH03C_32 [69] }\end{array}$ \\
\hline $\mathrm{P} \_$GFP_SpoTH_RFP & Fig. 21-b & P_GFP_SpoTH with luxR-Plux-RFP added from MBP-1.0 [1] \\
\hline P_GFP_SpoTH_dCas9 & Fig. 21-c & P_GFP_SpoTH_RFP with RFP replaced by dCas9 from pdCas9_OP in [50] \\
\hline $\mathrm{P} \_\mathrm{OL}$ & Fig. 21-d & P_GFP_SpoTH_RFP with TetR-pTet-SpoTH removed \\
\hline $\mathrm{P} \_$IFFL_1 & Fig. 21-e & P_OL with RBS 1-SpoTH directly downstream of RFP \\
\hline $\mathrm{P}_{\text {__IFFL_2 } 2}$ & Fig. 21-e & P_OL with RBS 2-SpoTH directly downstream of RFP \\
\hline $\mathrm{P} \_$IFFL_3 & Fig. 21-e & P_OL with RBS 3-SpoTH directly downstream of RFP \\
\hline $\mathrm{P} \_$IFFL_4 & Fig. 21-e & P_OL with RBS 4-SpoTH directly downstream of RFP \\
\hline P_Control & Fig. 21-f & $\begin{array}{c}\text { P_IFFL_2 but SpoTH replaced by CJB (cjBlue H197S [60]) } \\
\text { The CJB DNA is codon optimized for E. coli }\end{array}$ \\
\hline $\mathrm{P} \_$weak_RFP & Fig. 21-d & P_OL but changed RFP RBS from B34 to RBS weak (MBP-0.006 in [1]) \\
\hline $\mathrm{P} \_$weak_RFP_SpoTH_1 & Fig. 21-e & P_IFFL_1 but replaced RFP RBS from B34 to RBS weak \\
\hline $\mathrm{P} \_$weak_RFP_SpoTH_2 & Fig. 21-e & P_IFFL_2 but changed RFP RBS from B34 to RBS weak \\
\hline P_weak_RFP_SpoTH_3 & Fig. 21-e & P_IFFL_3 but changed RFP RBS from B34 to RBS weak \\
\hline $\mathrm{P}_{\text {__weak_RFP_SpoTH_4 }}$ & Fig. 21-e & P_IFFL_4 but changed RFP RBS from B34 to RBS weak \\
\hline
\end{tabular}

Table 1: Description of plasmids used in this study

\begin{tabular}{|c|c|}
\hline Part & Sequence (5' to $\left.3^{\prime}\right)$ \\
\hline B32 & TACTAGAGTCACACAGGAAAGTACTAG \\
\hline B34 & TACTAGAGAAAGAGGAGAAATACTAG \\
\hline B34t & AATTCATTAAAGAGGAGAAAGGTACC \\
\hline RBS 2 & TGAGCGCCGCCAGGGACCACGC \\
\hline RBS 3 & CTCGACACACCCCCTATTAAAT \\
\hline RBS 4 & CAAACCTCTACCGTAGGATTCGTCATT \\
\hline RBS weak & TCGGCCCTATACGACTCTAAACGTCGATG \\
\hline $\mathrm{J} 23100$ & TTGACGGCTAGCTCAGTCCTAGGTACAGTGCTAGC \\
\hline $\mathrm{J} 23114$ & TTtATGGCTAGCTCAGTCCTAGGTACAATGCtAGC \\
\hline pTet & TCCCTATCAGTGATAGAGATTGACATCCCTATCAGTGATAGAGATACTGAGCAC \\
\hline pLacIQ & TGGTGCAAAACCTTTCGCGGTATGGCATGATAGCGCC \\
\hline Plux & ACCTGTAGGATCGTACAGGTTTACGCAAGAAAATGGTTTGTTATAGTCGAATAAA \\
\hline LuxR & 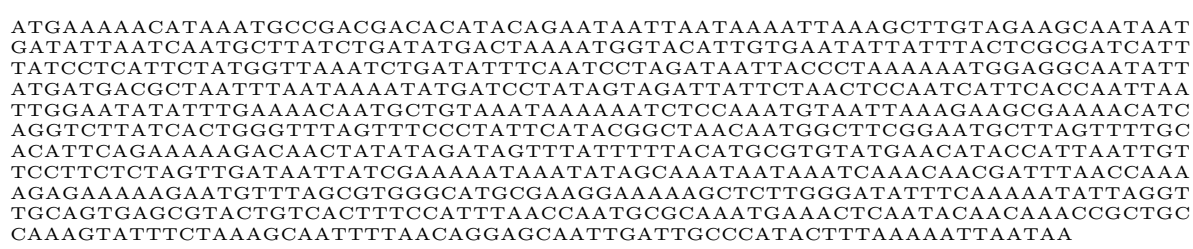 \\
\hline TetR & 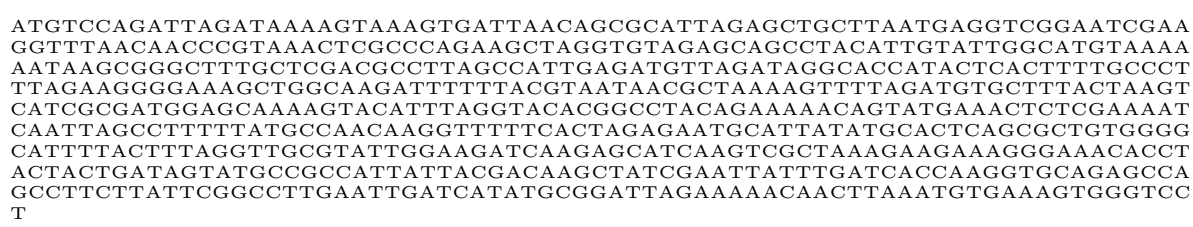 \\
\hline
\end{tabular}


bioRxiv preprint doi: https://doi.org/10.1101/2021.02.11.430724; this version posted June 24, 2021. The copyright holder for this preprint

(which was not certified by peer review) is the author/funder, who has granted bioRxiv a license to display the preprint in perpetuity. It is made available under aCC-BY-NC-ND 4.0 International license.

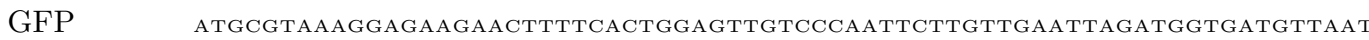
GGGCACAAATTTTCTGTCAGTGGAGAGGGTGAAGGTGATGCAACATACGGAAAACTTACCCTTAAATTT ATTTGCACTACTGGAAAACTACCTGTTCCATGGCCAACACTTGTCACTACTTTCGGTTATGGTGTTCAA
TGCTTTGCGAGATACCCAGATCATATGAACAGCATGACTTTTCAAGAGTCCATGCCGGAGGTAT GTACAGGAAAGAACTATATTTTTCAAAGATGACGGGAACTACAAGACACGTGCTGAAGTCAAGTTTGAA GGTGATACCCTTGTTAATAGAATCGAGTTAAAAGGTATTGATTTTAAAGAAGATGGAAACATTCTTGGA CACAAATTGGAATACAACTATAACTCACACAATGTATACATCATGGCAGACAAACAAAAGAATGGAATC AAAGTTAACTTCAAAATTAGACACAACATTGAAGATGGAAGCGTTCAACTAGCAGACCATTATCAACAA AATACTCCAATTGGCGATGGCCCTGTCCTTTTACCAGACAACCATTACCTGTCCACACAATCTGCCCTTT
CGAAAGATCCCAACGAAAAGAGAGACCACATGGTCCTTCTTGAGTTTGTAACAGCTGCTGGGATTACAC ATGGCATGGATGAACTATACAAATAATAA

RFP ATGGCTTCCTCCGAAGACGTTATCAAAGAGTTCATGCGTTTCAAAGTTCGTATGGAAGGTTCCGTTAAC GGTCACGAGTTCGAAATCGAAGGTGAAGGTGAAGGTCGTCCGTACGAAGGTACCCAGACCGCTAAACTG AAAGTTACCAAAGGTGGTCCGCTGCCGTTCGCTTGGGACATCCTGTCCCCGCAGTTCCAGTACGGTTCC AAAGCTTACGTTAAACACCCGGCTGACATCCCGGACTACCTGAAACTGTCCTTCCCGGAAGGTTTCAAA TGGGAACGTGTTATGAACTTCGAAGACGGTGGTGTTGTTACCGTTACCCAGGACTCCTCCCTGCAAGAC GGTGAGTTCATCTACAAAGTTAAACTGCGTGGTACCAACTTCCCGTCCGACGGTCCGGTTATGCAGAAA AAAATGCGTCTGAAACTGAAAGACGGTGGTCACTACGACGCTGAAGTTAAAACCACCTACATGGCTAAA AAACCGGTTCAGCTGCCGGGTGCTTACAAAACCGACATCAAACTGGACATCACCTCCCACA
TACACCATCGTTGAACAGTACGAACGTGCTGAAGGTCGTCACTCCACCGGTGCTTAATAA

SpoTH

ATGTATCTGTTTGAAAGCCTGAATCAACTGATTCAAACCTACCTGCCGGAAGACCAAATCAAGCGTCTG CGGCAGGCGTATCTCGTTGCACGTGATGCTCACGAGGGGCAAACACGTTCAAGCGGTGAACCCTATATC ACGCACCCGGTAGCGGTTGCCTGCATTCTGGCCGAGATGAAACTCGACTATGAAACGCTGATGGCGGCG GTCGCCGAGCTGGTAGAGGGGGTGTCGAAACTTGATAAACTCAAGTTCCGCGATAAGAAAGAGCGCA GGCCGAAAACTTTCGCAAGATGATTATGGCGATGGTGCAGGATATCCGCGTCATCCTCATCAAACTTGC CGACCGTACCCACAACATGCGCACGCTGGGCTCACTTCGCCCGGACAAACGTCGCCGCATCGCCCGTGA AACTCTCGAAATTTATAGCCCGCTGGCGCACCGTTTAGGTATCCACCACATTAAAACCGAACTCGAAGA AAATTCGTCAGTTGCTGAAAAACCTCAAGCGTAATAA

CJB ATGCATCATCATCATCATCATGCGTCGAAAATCTCAGACAATGTACGTATTAAACTGTACATGGAAGGC ACCGTCAACAACCATCACTTCATGTGTGAAGCGGAAGGTGAAGGCAAGCCGTACGAAGGAACCCAGATG GAAAACATTAAAGTCACTAAAGGTGGCCCTCTTCCGTTCTCTTTCGACATTCTCACGCCGAACTGTCAG TATGGGTCAGTGGCAATCACCAAATATACCAGTGGCATCCCGGACTACTTTAAGCAGAGCTTTCCTGAG GGATTCACGTGGGAACGCACGACCATCTATGAAGACGGGGCCTACCTTACAACCCAACAAGAAACGAAG
CTTGACGGCAACTGCCTGGTTATAACATCAAGATTCTGGTTGCAATTCCCACCGAACGGCCCGGTG CTTGACGGCAACTGCCTGGTGTATAACATCAAGATTCTGGGTTGCAATTTCCCACCGAACGGCCCGGTG ATGCAGAAGAAAACACAGGGATGGGAACCCTGTTGCGAGATGCGTTATACACGTGATGGAGTATTGTGT
GGGCAGACACTTATGGCGCTCAATGTGCAGATGGGACCACCTCACTTGCCACTTACGCACGACGTAC GGGCAGACACTTATGGCGCTCAAATGTGCAGATGGGAACCACCTCACTTGCCACTTACGCACGACGTAC GTGAAAGTTAGCGAGAATGGAACTCTTTTCGAACAGCATGAATCCTCAGTGGCCCGCTATTGCCAAACG TGTCCTTCCAAACTCGGCCATAACTAATAA

dCas9 ATGGATAAGAAATACTCAATAGGCTTAGCTATCGGCACAAATAGCGTCGGATGGGCGGTGATCACTGAT GAATATAAGGTTCCGTCTAAAAAGTTCAAGGTTCTGGGAAATACAGACCGCCACAGTATCAAAAAAAAT CTTATAGGGGCTCTTTTATTTGACAGTGGAGAGACAGCGGAAGCGACTCGTCTCAAACGGACAGCTCGT AGAAGGTATACACGTCGGAAGAATCGTATTTGTTATCTACAGGAGATTTTTTCAAATGAGATGGCGAAA GTAGATGATAGTTTCTTTCATCGACTTGAAGAGTCTTTTTTGGTGGAAGAAGACAAGAAGCATGAACGT CGAAAAAAATTGGTAGATTCTACTGATAAAGCGGATTTGCGCTTAATCTATTTGGCCTTAGCGCATATG ATTAAGTTTCGTGGTCATTTTTTGATTGAGGGAGATTTAAATCCTGATAATAGTGATGTGGACAAACTA TTTATCCAGTTGGTACAAACCTACAATCAATTATTTGAAGAAAACCCTATTAACGCAAGTGGAGTAGAT GCTAAAGCGATTCTTTCTGCACGATTGAGTAAATCAAGACGATTAGAAAATCTCATTGCTCAGCTCCCC GGTGAGAAGAAAAATGGCTTATTTGGGAATCTCATTGCTTTGTCATTGGGTTTGACCCCTAATTTTAAA TCAAATTTTGATTTGGCAGAAGATGCTAAATTACAGCTTTCAAAAGATACTTACGATGATGATTTAGAT AATTTATTGGCGCAAATTGGAGATCAATATGCTGATTTGTTTTTGGCAGCTAAGAATTTATCAGATGCT
ATTTTACTTTCAGATATCCTAAGAGTAAATACTGAATAACTAAGGCCCCTATCAGCTTCAATGATTA
AACGCTACGATGAACATCATCAAGACTTGACTCTTTTAAAAGCTTAGTTCGACAACACTTCCAGAAA AACGCTACGATGAACATCATCAAGACTTGACTCTTTTAAAAGCTTTAGTTCGACAACAACTTCCAGAAA
AGTATAAAGAAATCTTTTTGATCAATCAAAAAACGGATATGCAGGTTATATTGATGGGGGAGCTACC AGTATAAAGAAATCTTTTTTGATCAATCAAAAAACGGATATGCAGGTTATATTGATGGGGGAGCTAGCC
AAGAAGAATTTATAAATTATCAAACCAATTTAGAAAAAAGGATGTACTGAGGATTATTGGTGA AAGAAGAATTTTATAAATTTATCAAACCAATTTTAGAAAAAATGGATGGTACTGAGGAATTATTGGTGA
AACTAAATCGTGAAGATTTGCTGCGCAAGCAACGGACCTTTGACAACGGCTCTATTCCCCATCAAATTC AACTAAATCGTGAAGATTTGCTGCGCAAGCAACGGACCTTTGACAACGGCTCTATTCCCCATCAAATTC AGAAGATTGAAAAAATCTTGACTTTTCGAATTCCTTATTATGTTGGTCCATTGGCGCGTGGCAATAGTC GTTTTGCATGGATGACTCGGAAGTCTGAAGAAACAATTACCCCATGGAATTTTGAAGAAGTTGTCGATA AAGGTGCTTCAGCTCAATCATTTATTGAACGCATGACAAACTTTGATAAAAATCTTCCAAATGAAAAAG TACTACCAAAACATAGTTTGCTTTATGAGTATTTTACGGTTTATAACGAATTGACAAAGGTCAAATATG TTACTGAAGGAATGCGAAAACCAGCATTTCTTTCAGGTGAACAGAAGAAAGCCATTGTTGATTTACTCT TCAAAACAAATCGAAAAGTAACCGTTAAGCAATTAAAAGAAGATTATTTCAAAAAAATAGAATGTTTTG ATAGTGTTGAAATTTCAGGAGTTGAAGATAGATTTAATGCTTCATTAGGTACCTACCATGATTTGCTAA AAATTATTAAAGATAAAGATTTTTTGGATAATGAAGAAAATGAAGATATCTTAGAGGATATTGTTTTAA CATTGACCTTATTTGAAGATAGGGAGATGATTGAGGAAAGACTTAAAACATATGCTCACCTCTTTGATG ATAAGGTGATGAAACAGCTTAAACGTCGCCGTTATACTGGTTGGGGACGTTTGTCTCGAAAATTGATTA ATGGTATTAGGGATAAGCAATCTGGCAAAACAATATTAGATTTTTTGAAATCAGATGGTTTTGCCAATC CTGGACAAGGCGATAGTTTACATGAACATATTGCAAATTTAGCTGGTAGCCCTGCTATTAAAAAAGGTA TTTTACAGACTGTAAAAGTTGTTGATGAATTGGTCAAAGTAATGGGGCGGCATAAGCCAGAAAATATCG TTATTGAAATGGCACGTGAAAATCAGACAACTCAAAAGGGCCAGAAAAATTCGCGAGAGCGTATGAAAC GAATCGAAGAAGGTATCAAAGAATTAGGAAGTCAGATTCTTAAAGAGCATCCTGTTGAAAATACTCAAT TGCAAAATGAAAAGCTCTATCTCTATTATCTCCAAAATGGAAGAGACATGTATGTGGACCAAGAATTAG
ATATTAATCGTTTAAGTGATTATGATGTCGATGCCATTGTTCCACAAAGTTCCTTAAAGACGATTCAA ATATTAATCGTTTAAGTGATTATGATGTCGATGCCATTGTTCCACAAAGTTTCCTTAAAGACGATTCAA TAGACAATAAGGTCTTAACGCGTTCTGATAAAAATCGTGGTAAATCGGATAACGTTCCAAGTGAAGAAG
TAGTCAAAAAGATGAAAAACTATTGGAGACAACTTCTAAACGCCAATTAATCACTCAACGTAATTTG ATAATTTAACGAAAGCTGAACGTGGAGGTTTGAGTGAACTTGATAAAGCTGGTTTTATCAAACGCCAAT TGGTTGAAACTCGCCAAATCACTAAGCATGTGGCACAAATTTTGGATAGTCGCATGAATACTAAATACG ATGAAAATGATAAACTTATTCGAGAGGTTAAAGTGATTACCTTAAAATCTAAATTAGTTTCTGACTTCC AAAAAGATTTCCAATTCTATAAAGTACGTGAGATTAACAATTACCATCATGCCCATGATGCGTATCTAA ATAAAGTTTATGATGTTCGTAAAATGATTGCTAAGTCTGAGCAAGAAATAGGCAAAGCAACCGCAAAAT ATTTCTTTTACTCTAATATCATGAACTTCTTCAAAACAGAAATTACACTTGCAAATGGAGAGATTCGCAA ACGCCCTCTAATCGAAACTAATGGGGAAACTGGAGAAATTGTCTGGGATAAAGGGCGAGATTTTGCCAC AGTGCGCAAAGTATTGTCCATGCCCCAAGTCAATATTGTCAAGAAAACAGAAGTACAGACAGGCGGATT CTCCAAGGAGTCAATTTTACCAAAAAGAAATTCGGACAAGCTTATTGCTCGTAAAAAAGACTGGGATCC AAAAAAATATGGTGGTTTTGATAGTCCAACGGTAGCTTATTCAGTCCTAGTGGTTGCTAAGGTGGAAAA AGGGAAATCGAAGAAGTTAAAATCCGTTAAAGAGTACTAGGGATCACAATTATGGAAAGAAGT TCCT ACTACCTAAATATAGTCTTTTTGAGTTAGAAAACGGTCGTAAACGGATGCTGGCTAGTGCCGGAGAATT ACAAAAAGGAAATGAGCTGGCTCTGCCAAGCAAATATGTGAATTTTTTATATTTAGCTAGTCATTATGA AGATGAGATTATTGAGCAAATCAGTGAATTTTCTAAGCGTGTTATTTTAGCAGATGCCAATTTAGATAA AGTTCTTAGTGCATATAACAAACATAGAGACAAACCAATACGTGAACAAGCAGAAAATATTATTCATTT ATTTACGTTGACGAATCTTGGAGCTCCCGCTGCTTTTAAATATTTTGATACAACAATTGATCGTAAACG
ATATACGTCTACAAAAGAATTTTAGATGCCACTCTTATCCATCAATCCATCACTGGTCTTTATGAACA CGCATTGATTTGAGTCAGCTAGGAGGTGACTAA 


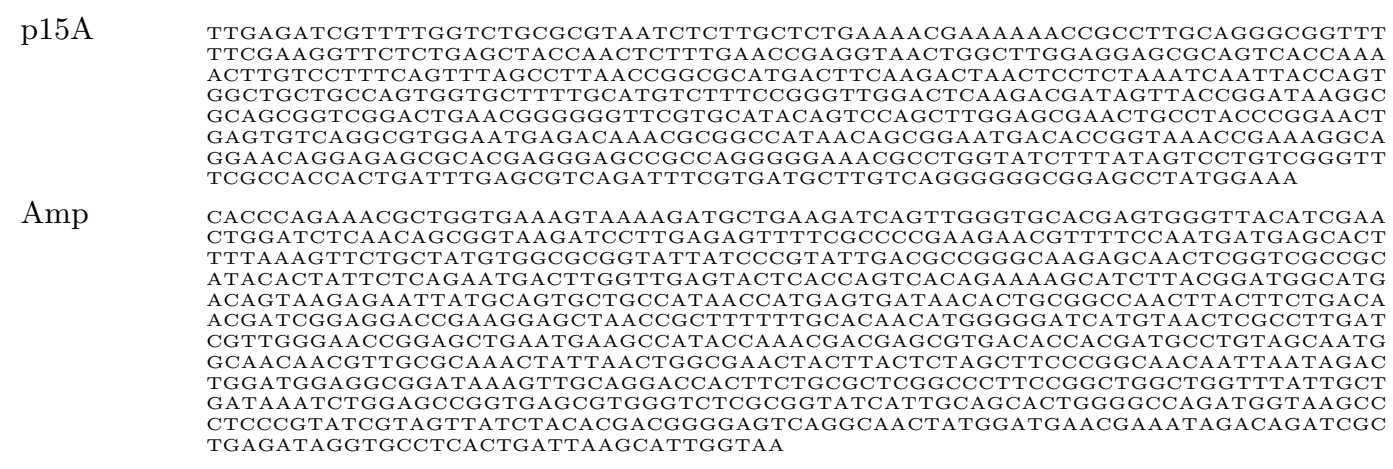

Table 2: Essential DNA sequences used in this study.

\section{Supplementary note 1}

We model mRNA $(\mathrm{m})$ binding to free ribosomes $(\mathrm{R})$ to produce the translation initiation complex $\mathrm{c}$, which is then translated to produce the protein $\mathrm{P}$ with elongation rate constant $\kappa$. The mRNA decays with rate constants $\delta$ and the protein dilutes with rate constant $\gamma$. The corresponding chemical reactions are:

$$
\emptyset \stackrel{\alpha_{\mathrm{m}}}{\longrightarrow} \mathrm{m}, \quad \mathrm{m} \stackrel{\delta}{\longrightarrow} \emptyset, \quad \mathrm{m}+\mathrm{R} \underset{\mathrm{d}}{\stackrel{\mathrm{a}}{\rightleftharpoons}} \mathrm{c} \stackrel{\kappa}{\longrightarrow} \mathrm{m}+\mathrm{R}+\mathrm{P}, \quad \mathrm{P} \stackrel{\gamma}{\longrightarrow} \emptyset
$$

where $\alpha_{m}$ is the production rate constant of the mRNA, $a$ and $d$ are the association and dissociation rate constant, respectively, between ribosomes and mRNA. The concentration of each species satisfies:

$$
\begin{aligned}
\frac{d m}{d t} & =\alpha_{m}-a R m+(d+\kappa) c-\delta m, \\
\frac{d c}{d t} & =a R m-(d+\kappa) c \\
\frac{d P}{d t} & =\kappa c-\gamma P .
\end{aligned}
$$

The ribosome-mRNA dynamics can be assumed to be fast relative to $\gamma$ [75] and thus the quasi-steady state [63] of (30) is given by

$$
m=\frac{\alpha_{m}}{\delta}, \quad c=\frac{m}{K} R,
$$

where $K=\frac{d+\kappa}{a}$. Thus, the reduced protein concentration dynamics are given by

$$
\frac{d P}{d t}=\underbrace{\frac{\kappa \alpha_{m}}{K \delta} R}_{\alpha_{P}}-\gamma P .
$$

where $\alpha_{P}$ is the protein production rate. If the protein is constitutively expressed, then $\alpha_{m}$ is constant and $\alpha_{P}$ is given by a constant $\left(\frac{\kappa \alpha_{m}}{K \delta}\right)$ multiplied by $R$, implying that the protein production rate is a proxy for free ribosome concentration.

\section{Supplementary note 2}

Our modeling framework (Modeling box 2 in the main text) suggests that we can tune the SpoTH RBS strength in the closed loop genetic circuit (express heterologous protein and SpoTH on the same mRNA) to minimize the sensitivity of free ribosomes on heterologous protein expression. Therefore, we created a SpoTH RBS library: RBS 1, RBS 2, RBS3, and RBS 4, to test on the closed loop circuit. In this section we characterize the relative strength of the library in the configuration where SpoTH is expressed on the same mRNA as RFP (placed upstream of SpoTH). We show that the strength of the RBS increases in the following order: RBS 1, RBS 2, RBS 3, and RBS 4.

The RBS strength is dependent on the upstream and downstream sequences of the RBS [76, 77], therefore we characterize the SpoTH RBS library with RFP upstream of SpoTH so that the results are applicable to the closed loop controller (Fig. 4-d in the main text). However, we decrease the RBS strength of RFP by several fold (MBP 0.006 in [1]) such that the amount of ribosomes it sequesters are negligible (relative to SpoTH actuation) and thus the change in ribosome concentration when expressing the mRNA with both the weak RFP RBS and SpoTH, is identical to SpoTH in isolation. The construct used to characterize the SpoTH RBS library is shown in Fig.20-a.

Increasing the RBS strength implies that for a fixed amount of SpoTH mRNA, more ribosomes are recruited to translate the mRNA and thus more SpoTH protein is produced. Therefore, less SpoTH mRNA is needed to actuate as 
the SpoTH RBS strength increases. This implies that when expressing SpoTH using the construct shown in Fig.20-a, less AHL is needed to see an actuation of GFP production rate and growth rate as the SpoTH RBS increases. The GFP production rate and growth rate data are shown in Fig.20-b and Fig.20-c, respectively, when expressing SpoTH using the genetic circuit in Fig.20-a with lactose as the carbon source. We observe that for the list: RBS 1, RBS 2, RBS 3, and RBS 4, that the amount of AHL needed to actuate the GFP production rate and growth rate decreases. Thus, based on our physical intuition, it implies that the RBS strength should have an increasing order of: RBS 1, RBS 2, BS 3, and RBS 4. The same trend is observed in Fig.20-d and Fig.20-e when using glycerol as the carbon source.

Our physical intuition that increasing the SpoTH RBS strength implies that less mRNA is needed to see an actuation on free ribosomes, can be made mathematically precise using the actuator model (20), which relates free ribosome concentration to SpoTH expression. From the fact that

$$
\bar{c}_{s}=m_{s} \bar{R} / K_{s}
$$

where $m_{s}$ is the SpoTH mRNA and $K_{S}$ is inversely proportional to the SpoTH RBS strength, to specify $\bar{c}_{s}$ we need to know the value $\bar{R}$. Therefore, we need to specify specify $\bar{c}_{e}(R)$. We assume that $\bar{c}_{e}(R)$ has a form similar to that of $\bar{c}_{s}$, then for $q$ different endogenous genes expressing mRNA, $\bar{c}_{e}(R)=\sum_{i=1}^{q} \frac{m_{e, i}}{K_{e, i}} \bar{R}$, where for gene $i$, $m_{e, i}$ is the endogenous mRNA concentration and $K_{e, i}$ is the effective dissociation constant of endogenous mRNA with ribosomes. In this cases, $\bar{c}_{e}(R)$ satisfies all of the assumptions stated in the main text Modeling Box 2 and furthermore, $\bar{R}=\frac{\bar{z}(R)}{1+\sum_{i=1}^{q} \frac{m_{e, i}}{K_{e, i}}}$. Let $m_{s}^{*}=\frac{m_{s}}{1+\sum_{i=1}^{q} \frac{m_{e, i}}{K_{e, i}}}$ and thus (6) now reads:

$$
\bar{z}_{0}+\left(1-\bar{z}_{0}\right) f\left(\bar{z} m_{s}^{*} / K_{s} / \epsilon, \bar{z}_{0}\right)=\bar{z}+\bar{z} m_{s}^{*} / K_{s}
$$

The results from simulating (32) are shown in Fig.21. We observe that increasing the RBS strength (decrease $K_{s}$ ) the amount of SpoTH mRNA $\left(m_{s}^{*}\right)$ needed to actuate $\bar{z}$ decreases.

\section{Supplementary note 3}

The SpoTH gene sequence was constructed based on the BssHII digestion and re-ligation of the spoT gene (pGN19 in [47]), which was shown to only have ppGppasse activity. The digestion and re-ligation of spoT using BssHII introduces a frameshift following the 206 codon and consequently a premature stop codon after the 217 codon. Therefore, the SpoTH sequence only contains the first 217 condons of the product of re-ligating and digesting spoT using BssHII. Finally we modified the initial codon of the endogenous spoT gene from TTG to ATG. The full SpoTH sequence is shown in SI Table 2.

\section{Supplementary note 4}

From the fact that

$$
\bar{c}_{y}=m_{y} \bar{R} / K_{y}, \quad \bar{c}_{s}=m_{s} \bar{R} / K_{s},
$$

to specify $\bar{c}_{y}$ and $\bar{c}_{s}$ we need to know the value $\bar{R}$. Therefore, we need to specify specify $\bar{c}_{e}(R)$. We assume that $\bar{c}_{e}(R)$ has a form similar to that of $\bar{c}_{s}$, then for $q$ different endogenous genes expressing mRNA, $\bar{c}_{e}(R)=\sum_{i=1}^{q} \frac{m_{e, i}}{K_{e, i}} \bar{R}$, where for gene $i, m_{e, i}$ is the endogenous mRNA concentration and $K_{e, i}$ is the effective dissociation constant of endogenous mRNA with ribosomes. In this cases, $\bar{c}_{e}(R)$ satisfies all of the assumptions stated in the main text Modeling Box 2 and furthermore, $\bar{R}=\frac{\bar{z}(R)}{1+\sum_{i=1}^{q} m_{e, i}}$. Let $J_{s}=\frac{m_{s} / K_{s}}{1+\sum_{i=1}^{q} \frac{m_{e, i}}{K_{e, i}}}$ and $J_{y}=\frac{m_{y} / K_{y}}{1+\sum_{i=1}^{q} \frac{m_{e, i}}{K_{e, i}}}$ and thus (6) now reads:

$$
\bar{z}_{0}+\left(1-\bar{z}_{0}\right) f\left(\bar{z} J_{s} / \epsilon, \bar{z}_{0}\right)=\bar{z}+\bar{z} J_{s}+\bar{z} J_{y}
$$

Note that $J_{s}$ and $J_{y}$ are a meausre of the amount of SpoTH mRNA and y mRNA concentration, respectively. The parameters used to simulate $(33)$ are $\bar{z}_{0}=0.25, \epsilon=0.13, J_{s}=0,0.10,0.15,0.21,0.26,0.3$, and vary $J_{y}$ between 0 to 3.2 for each value of $J_{s}$. 
bioRxiv preprint doi: https://doi.org/10.1101/2021.02.11.430724; this version posted June 24, 2021. The copyright holder for this preprint (which was not certified by peer review) is the author/funder, who has granted bioRxiv a license to display the preprint in perpetuity. It is made available under aCC-BY-NC-ND 4.0 International license.

\section{a}

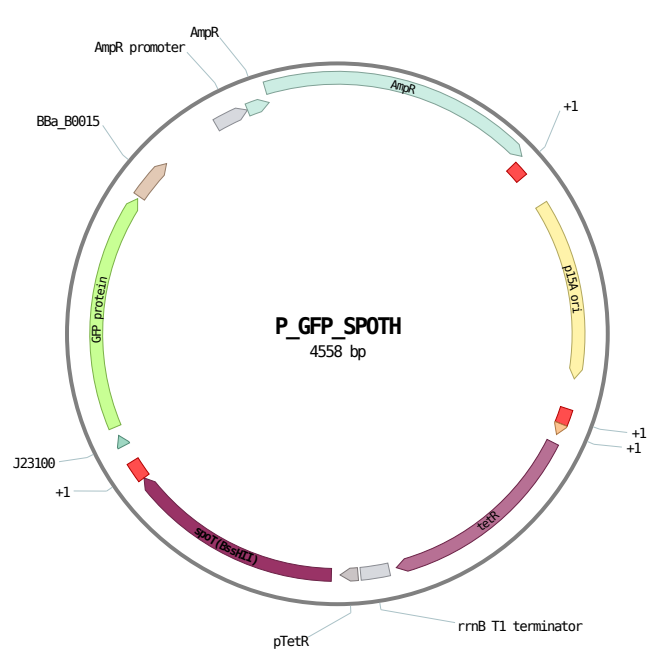

C

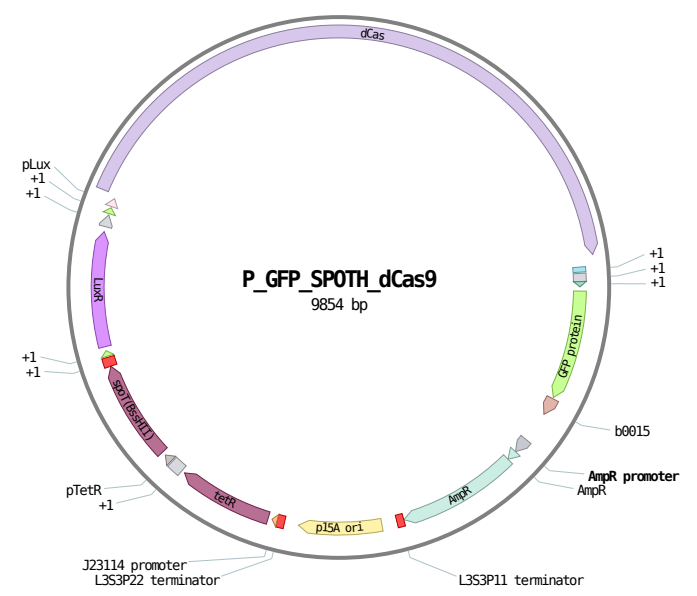

$\mathbf{e}$

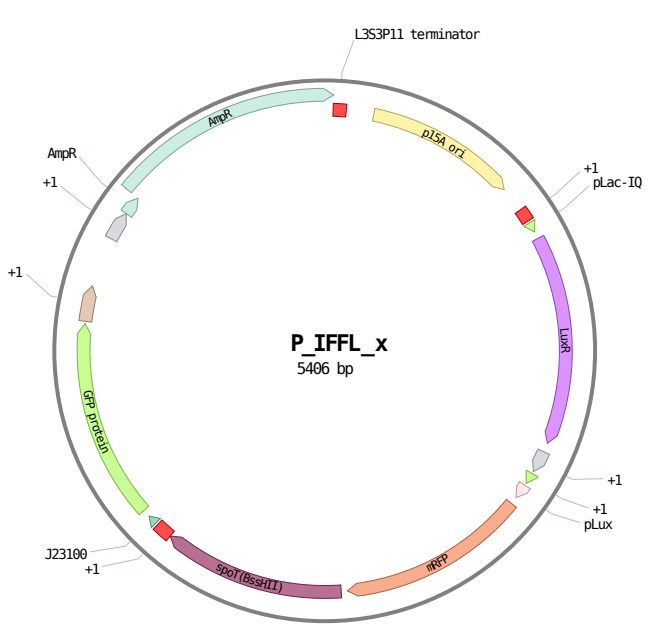

b

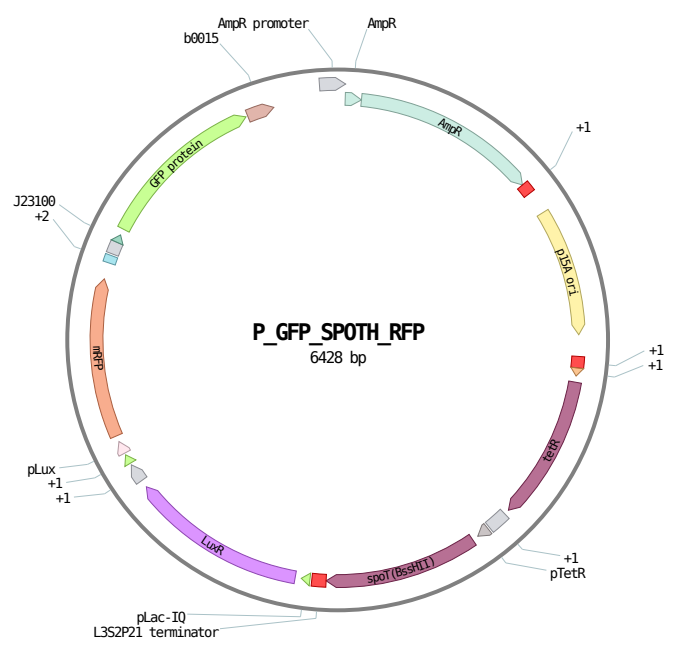

d

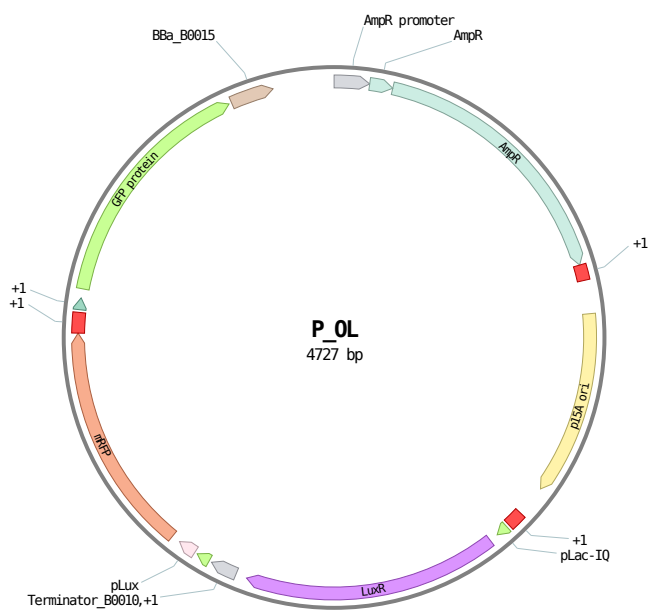

$\mathbf{f}$

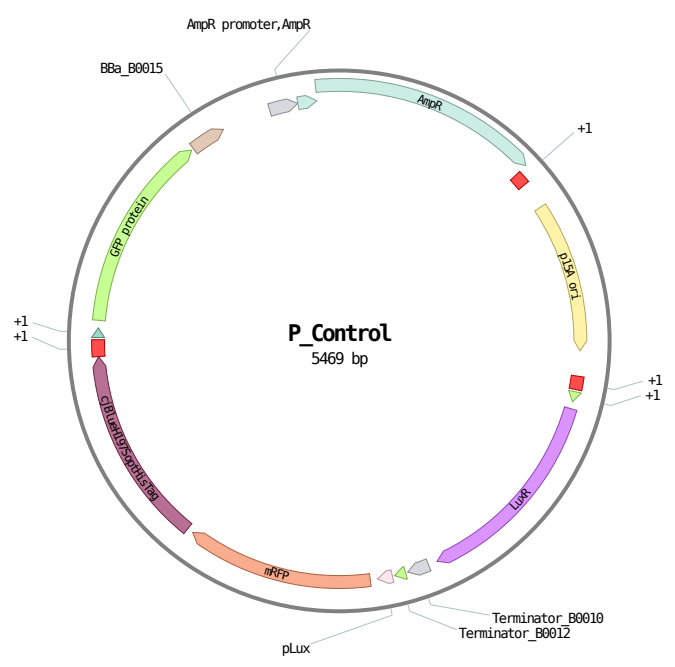

Figure 19: Plasmid maps The plasmid maps were prepared by the Benchling Life Sciences R\&D platform. (a) P_GFP_SpoTH (b) P_FP_SpoTH_RFP (c) P_GFP_SpoTH_dCas9 (d) P_OL (e) P_IFFL_x (f) P_Control 
bioRxiv preprint doi: https://doi.org/10.1101/2021.02.11.430724; this version posted June 24, 2021. The copyright holder for this preprint (which was not certified by peer review) is the author/funder, who has granted bioRxiv a license to display the preprint in perpetuity. It is made available under aCC-BY-NC-ND 4.0 International license.

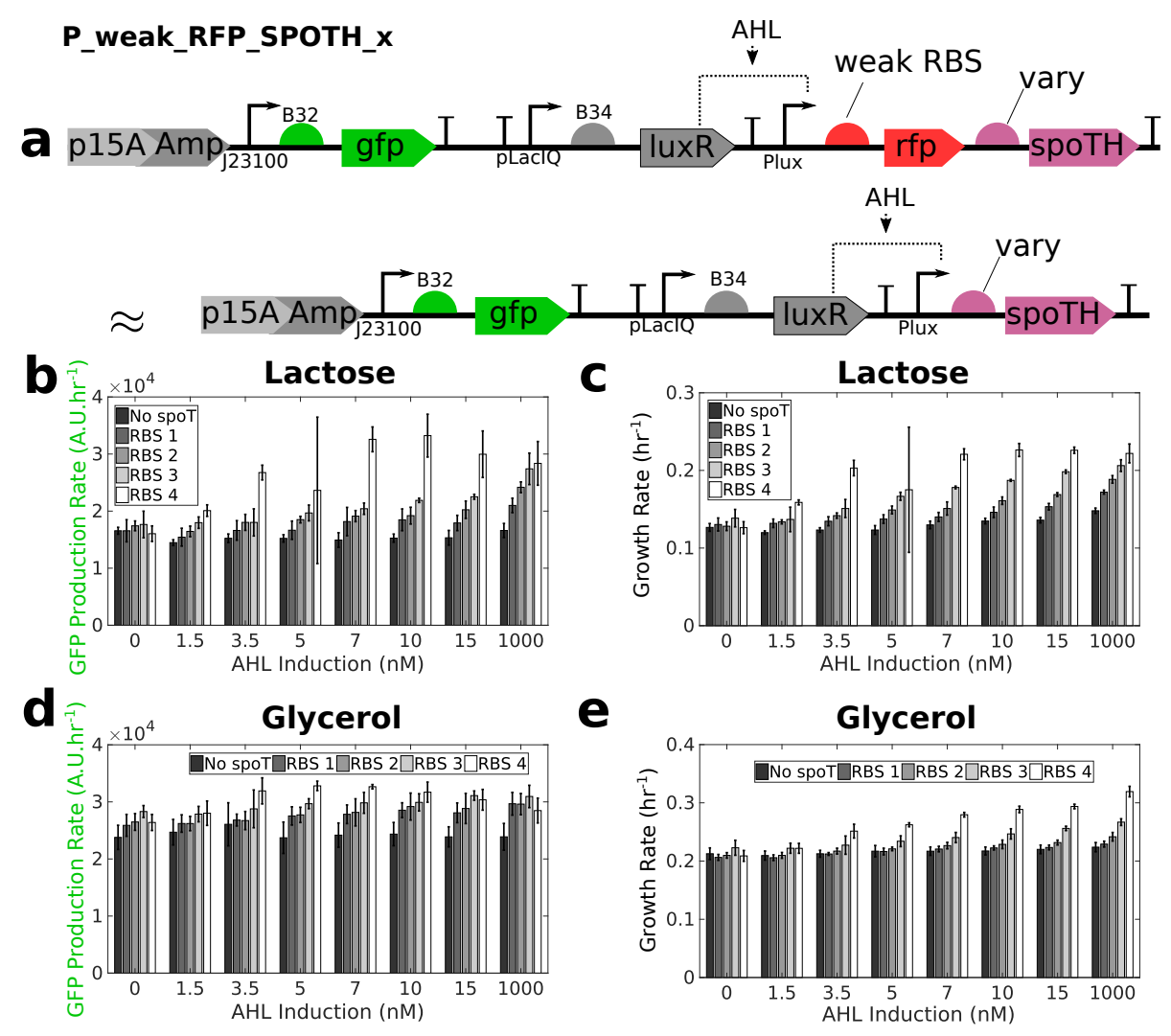

Figure 20: Characterizing the SpoTH RBS library strengths (a) The P_weak_RFP_SPOTH_x genetic construct used to characterize the SpoTH RBS library. This construct is identical to Fig. 4$\mathrm{d}\left(\mathrm{P} \_\right.$IFFL_x $)$in the main text, but with a very weak RFP RBS strength. The genetic construct P_weak_RFP (identical to P_OL with weak RBS for RFP) corresponds to "No SpoTH" in the legends. Plasmid description, plasmid map, and essential DNA sequences are provided in SI section Plasmid maps and DNA sequences. (b) For lactose as the carbon source, the GFP production rate as SpoTH is expressed (increase AHL) for the RBS library. (c) For lactose as the carbon source, the growth rate as SpoTH is expressed (increase AHL) for the RBS library. (d) For glycerol as the carbon source, the GFP production rate as SpoTH is expressed (increase AHL) for the RBS library. (e) For glycerol as the carbon source, the growth rate as SpoTH is expressed (increase AHL) for the RBS library. For all data, error bars represent standard deviation from at least four replicates (two biological replicates each with two technical replicates). Data are shown as the mean \pm one standard deviation $(\mathrm{N}=4$, two biological replicates each with two technical replicates). The complete experimental protocol is provided in the Materials and Methods section.

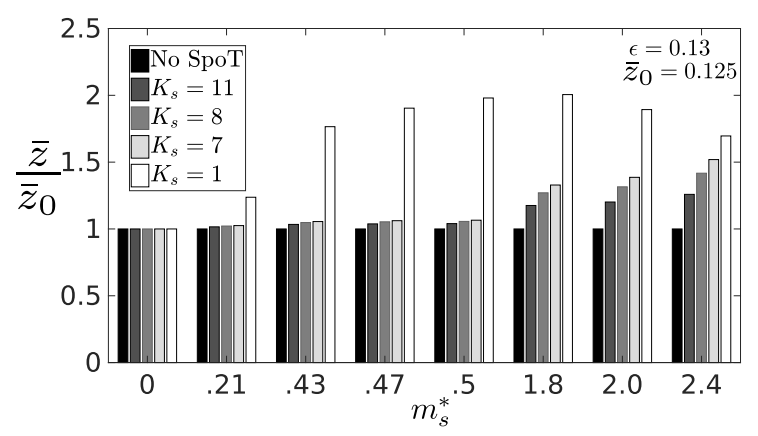

Figure 21: SpoTH expression with several RBS strengths The normalized measure of free ribosome concentration ${ }^{\overline{ }} z / \bar{z}_{0}$ predicted by (32) as the normalized SpoTH mRNA $m_{s}^{*}$ and SpoTH RBS strength $\left(1 / K_{s}\right)$ are varied. The simulation parameters are $\epsilon=0.13$ and $\bar{z}_{0}=0.125$. The "No SpoT" bars correspond to $\bar{z} / \bar{z}_{0}=1$ for all $m_{s}^{*}$ values. 